\title{
Cognitive task avoidance correlates with fatigue-induced performance decrement but not with subjective fatigue
}

\author{
Charles-Etienne Benoit ${ }^{\mathrm{a}, 1}$, Oleg Solopchuk ${ }^{\mathrm{a}, \mathrm{b}, 1}$, Guillermo Borragán ${ }^{\mathrm{a}, \mathrm{c}}$, Alice Carbonnelle ${ }^{\mathrm{a}}$, Sophie Van \\ Durme $^{a}$, Alexandre Zénon ${ }^{a, b}$
}

\footnotetext{
${ }^{a}$ Institute of Neuroscience, Université catholique de Louvain (UCL), B-1200 Brussels, Belgium

${ }^{b}$ INCIA, 33076, Bordeaux, France

${ }^{\mathrm{C}}$ Centre de Recherches en Cognition et Neurosciences and UNI - ULB Neurosciences Institute, Université Libre de Bruxelles (ULB), Belgium, Brussels, Belgium

${ }^{1}$ The first two authors contributed equally to this work
}

Corresponding author:

Alexandre Zénon, alexandre.zenon@u-bordeaux.fr 


\begin{abstract}
Mentally demanding tasks feel effortful and are usually avoided. Furthermore, prolonged cognitive engagement leads to mental fatigue, consisting of subjective feeling of exhaustion and decline in performance. Despite the intuitive characterization of fatigue as an increase in subjective effort perception, the effect of fatigue on effort cost has never been tested experimentally. To this end, sixty participants in 2 separate experiments underwent a forcedchoice working memory task following either a fatigue-inducing (i.e. cognitive task involving working memory, conflict and switch costs) or a control manipulation. We measured fatigue in terms of subjective feeling and performance decrement and assessed effort in terms of subjective perception and task avoidance. Surprisingly, we found that task avoidance did not systematically change following the fatigue manipulation. However, variations in task avoidance correlated with fatigue-induced performance decline, while the other measures of fatigue and effort were unrelated to each other. Our findings indicate that subjective fatigue develops independently of effort perception and suggest an "anticipatory regulation" model in which fatigue urges subjects to stop in anticipation of possible, future adverse consequences.
\end{abstract}

Keywords: Fatigue, Cognitive Effort, Neuroeconomics, N-Back task

\title{
1. Introduction
}

Demanding cognitive activities are typically aversive and lead to the percept of effort (Inzlicht et al., 2015; Westbrook and Braver, 2015). The subjective, introspective dimension of cognitive effort is assessed by means of self-report questionnaires, such as the NASA task-load index (Hart and Staveland, 1988), but cognitive effort can also be evaluated behaviorally by assessing task avoidance. This can be done by asking participants to choose between alternative options involving tasks of varied difficulty, associated with rewards of varied magnitude and by using neuroeconomic models to evaluate the effort cost of each task, i.e. how much performing the task discounts the associated reward (Hosking et al., 2014; Westbrook et al., 2013).

While the existence of a cost to cognition appears intuitively obvious, its origin remains unknown (Kurzban et al., 2013; Shenhav et al., 2017; Westbrook and Braver, 2015). What is clear, however, is that prolonged cognitive engagement leads to the subjective feeling of fatigue (Ackerman and Kanfer, 2009; Campagne et al., 2004; Deluca, 2005) and can also deteriorate performance (Holtzer et al., 2011; Schwid et al., 2003; Tanaka, 2015), sometimes referred to as objective fatigue (Bailey et al., 2007). The nature of these behavioral manifestations of cognitive 
fatigue is diverse, including disruption of inhibition mechanisms (Kato et al., 2009), planning (Lorist, 2008; Lorist et al., 2000; van der Linden et al., 2003), processing of new information (Massar et al., 2010), attention (Boksem et al., 2006; Dorrian et al., 2007; Holtzer et al., 2011; Lim et al., 2010), working memory (Gergelyfi et al., 2015) or task switching (Borragán et al., 2017). Like cognitive effort, the origin of cognitive fatigue remains unclear. Some have proposed that cognitive fatigue would result from metabolic depletion (Christie and Schrater, 2015; Fairclough and Houston, 2004; Gailliot and Baumeister, 2007) but this view has been strongly disputed (Carter et al., 2015; Vadillo et al., 2016). Other hypotheses include the spreading of local sleep patterns in the brain (Krueger et al., 2008; Vyazovskiy et al., 2011), the buildup of a decision signal aimed at limiting the opportunity cost of engaging cognitive resources in the same task (Kurzban et al., 2013) or the extended use of high-consuming strategies aimed at maintaining performance (i.e. Compensatory Control Model (CCM); Hockey, 2013, 1997).

One puzzling and pervasive observation in the fatigue literature is that the subjective dimension typically fails to correlate with the behavioral consequences of fatigue (Deluca, 2005). According to Hockey's influential CCM model (Hockey, 2013, 1997), this lack of relation between the subjective and behavioral dimensions would be explained by the fact that subjective fatigue is the manifestation of compensatory resource mobilization, needed to maintain performance despite the progressive deterioration of capacity induced by fatigue. Therefore, initially, subjective fatigue would predominate, while behavioral performance decrement would occur later on in the building up of fatigue. Subjective feelings of fatigue would appear as an indication that the resources needed to accomplish the ongoing demands are at risk or no longer available. In support of this view, subjective task aversion has been shown to increase concomitantly to time on task, even though it is unclear whether this increase is actually caused by fatigue (Lorist et al., 2000). Along the same line, cognitive fatigue increases blood pressure during cognitive task execution, but this effect has complex dependence on the likelihood and importance of the outcome (Stewart et al., 2009; Wright et al., 2007). Finally, neurophysiological activity suggestive of increased engagement during the compensatory phase of cognitive fatigue has also been recently reported (Wang et al., 2016).

Phenomenologically, subjective fatigue is often reported as increased aversion for cognition and increased percept of effort (Arai, 1912). In addition, the CCM assumes that subjective fatigue should be accompanied with increased percept of cognitive effort, and presumably increased task avoidance caused by the compensatory engagement of resources. To the extent of our knowledge, the effect of fatigue on these behavioral correlates of effort has never been 
experimentally assessed. We hypothesized that subjective fatigue would increase the cost of effort: a fatigued person would feel more effort performing a task of equivalent difficulty and would increase their preference for low-demand tasks. Sixty participants engaged in 2 separate experiments in which subjective effort and task avoidance were measured following either a fatigue-inducing or control manipulation. We evaluated fatigue both in its subjective dimension and in its behavioral manifestation as performance decline, and controlled for other variables such as sleepiness, arousal and motivation. We found that fatigue-related performance decrement correlated with task avoidance, while subjective feelings of fatigue and effort were unrelated to each other.

\section{Experiment 1}

\subsection{Materials and methods}

\subsubsection{Participants}

Thirty right-handed healthy participants took part in Experiment 1 (27 F, 3 M, Age: 22.8 +- 2.5, mean, SD), receiving monetary compensation for their participation (70 - 100€). Each experiment was performed over the span of three consecutive days. This included training on day 1 and fatigue/control manipulation followed by the working memory task on the experimental days 2 and 3, all occurring at the same time during the day, to control for circadian effects between sessions. All participants reported a normal or corrected-to-normal vision, had no auditory impairments or neurological disorders. Experiments were carried out according to the Declaration of Helsinki and were approved by the Ethics Committee of the Université catholique de Louvain. All participants provided a written informed consent.

\subsubsection{Subjective evaluation}

Participant's fatigue was assessed using the French version of the multidimensional fatigue inventory (MFI) (Gentile et al., 2003), composed of fifteen statements to be rated on a 5-point Likert scale (from "1 - Yes, that is true" to "5 - No, that is not true"). The answers to all questions were summed to obtain a Fatigue Index, in which lower score reflected higher fatigue. MFI was evaluated at the beginning and at the end of both experimental days.

In order to assess sleepiness, we used the 9-point Karolinska sleepiness scale (KSS) (Åkerstedt et al., 2014; Åkerstedt and Gillberg, 1990). Participants had to select a number 
corresponding to their level of sleepiness (from $1=$ extremely alert to $9=$ very sleepy, fighting against sleep). KSS was evaluated simultaneously with the MFI.

We also evaluated participants' intrinsic motivational state using intrinsic motivation inventory (IMI, http://selfdeterminationtheory.org/intrinsic-motivation-inventory/) (Ryan and Deci, 2000). The subjects provided the ratings on a 7-point Likert scale (from "Not at all true" to "Very true"), thus, higher scores represented higher task interest/enjoyment.

Subjective effort was evaluated using the National Aeronautics and Space Administration Task Load Index (NASA-TLX) rating scale (Hart and Staveland, 1988). It is divided in six subscales: mental demand, physical demand, temporal demand, performance, effort and frustration level. In the present study, we focused only on mental demand, performance and effort. Since the concepts of mental demand and effort were difficult to disentangle for participants, and since the scores strongly correlated with each other, we took the average of the mental demand and effort scores as an index of subjective effort cost. The participants had to score each of the items on a 20-point scale presented directly on screen. Participants completed the NASA-TLX after each block of the working memory task on both experimental days.

\subsubsection{Fatigue/Control manipulation}

On experimental days, participants underwent $2 \mathrm{~h}$ of either fatigue or control manipulation (see Figure 1). Fatigue was induced by performing a conflict-switch-working memory (CSWm) task in which subjects had to make decision on the basis of conflicting sources of information (i.e. a variant of the Stroop task (MacLeod, 1991)), an approach that has been used widely as a way of promoting cognitive fatigue (Moeller et al., 2012; Pageaux et al., 2015; Wang et al., 2016). In addition, the CSWm task involved two different types of stimulus-response associations and participants had to switch regularly from one to the other, leading to switch costs, also known to promote fatigue (Lorist et al., 2000). Finally, the CSWm task required to maintain information in working memory, since the cue providing instruction for the task was given after the presentation of the stimulus. In the control session, participants watched emotionally neutral documentaries. The order of the sessions (fatigue/control) was counterbalanced between all participants. In the CSWm task, participants were instructed to be as accurate and fast as possible. The time between the cue onset and the initiation of the next trial was adapted to the performance of each participant, according to the following rule:

$$
\mathcal{M}=\sum_{t=1}^{T} \mathcal{M} \times 0.75+\operatorname{accuracy}(t) \times 0.25
$$




$$
\text { responseTime }=\left\{\begin{array}{l}
\text { responseTime } \times 1.1 \text { if } \mathcal{M} \leq 0.8 \\
\text { responseTime } \times 0.9 \text { if } \mathcal{M}>0.8
\end{array}\right.
$$

with $T$ being the current trial number, responseTime being initialized to $1500 \mathrm{~ms}$ at the beginning of each block, $\mathcal{M}$ being intialized to zero, and accuracy(t) representing the accuracy of the response in trial $t$ (one if correct, zero if incorrect). responseTime was 300 ms minimum and $3000 \mathrm{~ms}$ maximum. This procedure ensured that the task remained challenging for all subjects, independently of their capacity. Each block contained a total of 368 trials, distributed equally between 2 different tasks. In the number task, participants were presented with two numbers, on either side of the fixation point (left and right), and had subsequently to report the location of either the largest in value or the largest in size, depending on an instruction cue. In the arrow task, one arrow was presented either above or below the fixation point and participants were to report either its position or direction, depending on the instruction cue. Trials were equally divided into congruent (e.g. large digit with large value or upwards pointing arrow located on the upper half of the screen) and incongruent (e.g. large digit with small value or upwards pointing arrow located on the lower half of the screen) trials. The participants completed 8 blocks in 2 hours. Each trial proceeded as follows: the fixation cross appeared in the middle of the screen for $1000 \mathrm{~ms}$, followed by $250 \mathrm{~ms}$ of stimuli presentation. After a delay, a blank screen appeared for 500-1000 ms (depending on the participant performance), and the instruction cue ("arrow" task: "direction" or "location"; "number" task: "value" or "size") was presented for $550 \mathrm{~ms}$. Participants had to answer within the cue presentation time, equal to the responseTime variable detailed above. The next trial began right after participants' response. The probability of task switches (defined as a change in either congruency or task instruction) decreased geometrically with each successive trial, such that $45 \%$ of trials involved a task switch, $26 \%$ followed one trial with the same task, while $12 \%, 8 \%, 5 \%$ and $2 \%$ had $2,3,4$ and 5 repetitions, respectively.

During the control session, participants watched animal documentaries on a computer for $2 \mathrm{~h}$. The documentaries were chosen according to their preference out of 7 different movies. Similar approaches have already been used in other experiments as a control condition (Badin et al., 2016; Van Cutsem et al., 2017; Zering et al., 2017).

\subsubsection{Effort discounting evaluation}

All tasks were implemented using version 3.0.9 of the Psychotoolbox (Brainard, 1997) in Matlab 7.5 (The MathWorks, Inc. Natick, Massachusetts, United States). Participants faced a 19-inch 
CRT screen with a refresh rate of $100 \mathrm{~Hz}$. The distance between the screen and the chin support was $58 \mathrm{~cm}$.

To evaluate effort and assess the consequences of fatigue, we used a variant of the auditory "N-back" working memory task, in which the subjects are required to judge whether the presented letter matches the one heard "N" letters before (Kirchner, 1958). Series of letters from the French alphabet, excluding F, J, M, P, W, Y, were presented aurally to participants. These letters were recorded from a young female voice and then normalized for consistency in pitch and volume. In each block, $25 \%$ of the letters were defined as targets, the others being considered as distractors. Task difficulty ' $N$ ' was calibrated individually during the training on the first day, and stayed constant during the whole experiment. The training included 15 blocks of increasing demand ( $N=1$ to 5 ), composed of 60 letters each. The letters were presented continuously while participants fixated the cross in the center of the screen. Response timeout was fixed to 1.5 seconds (indicated by change of fixation cross orientation), with an interstimulus interval of 2 to 3 seconds (uniformly distributed). A short pause was provided after every 5 blocks. The answers were collected via computer keyboard (distractor and target corresponded to button number ' 1 ' and '2' respectively). When the participant performance was greater than or equal to $70 \%$ (mean of the \% of targets detected and the \% of distractors correctly rejected) they progressed to the next level with a maximum difficulty level set to 5back, otherwise they repeated the same level once more. The maximal difficulty reached by the participants in the last block was then used for all subsequent experimental acquisitions (see Figure 2C).

Following the fatigue or control manipulation on experimental days, participants performed the $\mathrm{N}$-back tasks of different difficulty levels, presented in the context of a forced-choice paradigm, inspired from earlier studies (Westbrook et al., 2013). In order to avoid that participants' preference for difficulty-reward combinations would be influenced by their auto-evaluation of performance, rather than by effort perception, we defined task difficulty as the ratio of 1-back and N-back tasks in the block of 80 letters (actual ' $N$ ' depending on participants' performance during training, cfr. supra). These ratios are referred to as the block difficulty level (DL): \% of $N$ back: $\mathrm{DL}-1=25 \%, \mathrm{DL}-2=37.5 \%, \mathrm{DL}-3=50 \%$, DL-4 $=62.5 \%$, DL-5 = 75\%). The difficulty was represented visually using horizontal color bars (blue = 1-back; orange = n-back; see Figure 1). A fixed amount of $2 €$ was always associated with the DL-5 block while a variable amount, ranging between 0 and $2 €$ (with $0.20 €$ intervals) was offered for the easy option (i.e. DL- 1 to DL-4). Participants had to make the choice by pressing the right or left mouse button depending 
on whether they wanted to execute the task with the DL presented on the right or left part of the screen. The side of easy and difficult offers was pseudo-randomized for every participant. All possible difficulty pairings were evaluated for each possible reward level, which resulted in 44 forced-choices. Within these selections, participant had to perform the actual N-back task for 15 randomly selected choices. Any of the forced-choices could be selected for execution since the randomization did not take into account the proposed difficulty levels. Participants had to perform the selected block immediately following the choice. In each block, the accuracy (average percentage of target hits and distractor correct rejections) was averaged between both difficulty levels (1-back and N-back) to calculate the performance. This particular procedure ensured that, regardless of $\mathrm{DL}$, performance in the 1-back and in the N-back tasks had the same impact on the amount of money earned at the end of the block. For instance, if participants had average performance of $90 \%$ in the easy and $60 \%$ in the difficult task, their reward would be $75 \%$ of the proposed amount, irrespective of the relative proportion of easy and difficult trials (DL). This provided incentives for participants to disregard their relative performance in the easy and difficult tasks, and to consider only cognitive effort associated with the DL when making their choice. The remuneration was calculated by multiplying the performance by the reward attributed to the selected block (i.e. 0 to $2 €$ ). This information was provided on the screen at the end of each block. Prior to receiving performance feedback, participants had to answer the NASA-LTX questionnaire, presented on the computer screen.

\subsubsection{Statistical analyses}

We analyzed data with Matlab (The MathWorks, Inc., Natick, MA, USA) and performed statistical analyses with JASP (Version 0.8.2, JASP Team 2017). We performed both frequentist and Bayesian repeated-measure ANOVAs and Kendall correlations, to avoid undue influence of outliers. Results from the frequentist analyses are reported in terms of $p$-value (and $F$ statistics for the ANOVA), whereas Bayesian analyses resulted in Bayes Factors (BF). BF represents the ratio of the likelihood of two different models with, most classically, one assuming the alternative hypothesis and the other assuming the null hypothesis. Bayesian analysis was used in the present study because it provides a better estimate of effect reliability (BF) than the p-value. In addition, it provides the ability to evaluate the probability that the null hypothesis is true. Finally, it mitigates considerably the issue of multiple comparison (Gelman and Tuerlinckx, 2000). The BF can be interpreted on the basis of the table proposed in Kass and Raftery (1995): 
Evidence against $H_{0}$

Not worth more than a bare mention

Substantial

Strong

Decisive

For 1-way ANOVAs and correlation analyses, the $\mathrm{BF}_{10}$ is reported, which represents the comparison between the alternative and the null hypothesis. For 2-way ANOVAs, BF inclusion is reported instead. $\mathrm{BF}_{\text {inclusion }}$ is obtained by comparing the likelihood of the model including, or excluding a given effect.

The behavior of the participant in the choice task was analyzed with a computational model of effort-based decision making inspired from earlier studies (Zénon et al., 2016)

$$
\begin{gathered}
Q(\alpha, \beta, R, D)=\alpha\left[(1-\beta) \frac{R-100}{10}+\beta(D-1)\right] \\
\alpha=\frac{1}{e^{\text {temperature }}} \\
\beta=\frac{1}{1+e^{- \text {weight }}} \\
P(\text { easy|temperature, weight }, R, D)=\frac{1}{1+e^{-Q}}
\end{gathered}
$$

with temperature and weight as free model parameters. $R$ represented the amount of reward proposed for the difficult version of the task, and $D$ accounted for the difficulty level (ranges of $R$ and $D$ in the model corresponded to their range in the actual experiment). $Q$ represented an estimate of the net value of the proposed trial. $P$ (easy) indicated the probability of choosing the easy option, which was a function of the temperature and weight parameters and of the reward and difficulty conditions. The model was fit with multidimensional nonlinear minimization (Nelder-Mead, fminsearch) with ridge regularization.

The weight parameter can be interpreted as representing the relative impact of the difficulty level on the decisions of the participants, while the temperature parameter indicated how noisy their choices were. Increasing the weight parameter results in more effort-discounting (the 
difficulty parameter $D$ has more weight on the decision), while increasing the temperature parameter resulted in choices that were more stochastic (i.e. depended less on net value).

\subsection{Results}

CSWm task showed time-on-task increase in conflict and switch cost

Performance in the CSWm task was $92 \pm 3 \%, 93 \pm 2 \%, 45 \pm 2 \%$ and $80 \pm 3 \%$ (mean $\pm 95 \%$ confidence interval) for congruent/switch, congruent/no-switch, incongruent/switch and incongruent/no-switch trials, respectively, confirming that the task was challenging for the participants. We measured the difference in average performance block by block between congruent and incongruent conditions, either in switch or no-switch trials. We found that this incongruency effect increased over time in the switch (Bayesian t-test on Kendal correlation coefficients with block number; $\left.\mathrm{BF}_{10}=29637\right)$, but decreased in no-switch trials $\left(\mathrm{BF}_{10}=5.34\right)$. Conversely, we found that the effect of switch on accuracy increased also over time in the incongruent $\left(B F_{10}=19.66\right)$ but decreased in the congruent trials $\left(B F_{10}=28.80\right)$. We considered the increase in congruency and switch effect over time as potential indicators of fatigue buildup and correlated the corresponding correlation coefficients with the other subjective and behavioral measures (NASA scores, N-back performance scores, model parameters, MFI, IMI and $\mathrm{KSS})$. However, none of these correlations were significant $\left(\mathrm{BF}_{10}<3\right)$.

\section{CSWm session increases MFI and subjective effort but not task avoidance or performance}

This first analysis aimed at assessing the effect of the fatigue-inducing task on our different dependent variables. We performed a one-way repeated-measure ANOVA on the post-pre difference in MFI, KSS and IMI scores with SESSION as factor (control vs fatigue). There was no effect of SESSION on either KSS $\left(F(1,29)=2.188, p=0.15, B_{10}=0.759\right)$ or $\operatorname{IMI}(F(1,29)=1.965$, $p=0.172, B F_{10}=0.672$ ) and a small influence of SESSION on MFI (see Figure $3 A ; F(1,29)=5.075$, $p=0.032, B F_{10}=1.893$; i.e. significant according to frequentist statistics but anecdotal for Bayesian statistics). We then correlated these fatigue-related changes in subjective measures between each other. We found a very strong positive correlation between fatigue-control changes in MFI and KSS (Kendall's tau=0.508, $p<0.0001, B_{10}=283.292$ ), suggesting that the subjects failed to distinguish clearly the subjective feeling of fatigue from that of sleepiness. Finally, neither KSS nor MFI correlated with IMI (all $p>0.555$, all $\mathrm{BF}_{10}<2.33$ ), indicating that subjects' intrinsic motivation was not affected by fatigue manipulation, in accordance with previous studies (Gergelyfi et al., 2015). 
We then looked at the effect of fatigue and difficulty level on the NASA questionnaires. We found that the NASA subjective effort and performance scores changed as a function of the DIFFICULTY level (2-way RM-ANOVA: NASA effort: $F(4,116)=9.564, p<0.001, B_{\text {inclusion }}=4281$, NASA performance: $\left.F(4,116)=4.767, p=0.001, B_{\text {inclusion }}=17.9\right)$. In addition, NASA effort, but not NASA performance changed with SESSION (see Figure 3C; NASA effort: $F(1,29)=4.581$, $p=0.041, B F_{\text {inclusion }}=361$, NASA performance: $\left.F(1,29)=0.963, p=0.335, B F_{\text {inclusion }}=0.228\right)$. There was no SESSION $X$ DIFFICULTY interaction (NASA effort: $F(4,116)=0.180, p=0.949$, $B F_{\text {inclusion }}=0.025$, NASA performance: $\left.F(4,116)=0.163, p=0.956, B F_{\text {inclusion }}=0.027\right)$. There was no correlation between the fatigue-related changes in the two variables (Kendall's tau=-0.080, $\left.\mathrm{p}=0.547, \mathrm{BF}_{10}=0.284\right)$.

The temperature and weight parameters from the task avoidance models were also subjected to a one-way repeated-measure ANOVA with SESSION as factor (control vs fatigue). The weight parameter can be interpreted as an index of behavioral effort cost, while temperature indicates the level of randomness of participants' behavior. There was no change in either parameters between sessions (see Figure 2A, left panel, and Figure 3B; temperature: $F(1,29)=0.179$, $p=0.676, B F_{10}=0.285$, weight: $\left.F(1,29)=0.677, p=0.418, B F_{10}=0.353\right)$. There was a strong correlation between the fatigue-control changes in the two variables (Kendall's tau=0.526, $p<0.001, B F_{10}=704.0$ ). However, we noticed that many participants systematically picked the difficult task, irrespective of the reward value (13 subjects picked the difficult task in $100 \%$ of choices in at least one session). On average the percentage of choice of the difficult task was $86.4 \pm 2.7 \%$ and $87.4 \pm 2.6 \%$ for the fatigue and the control sessions, respectively (mean $\pm \mathrm{Cl}$ ).

In terms of fatigue-related changes in task performance, a 2-way RM-ANOVA on the logittransformed accuracy scores showed, besides the obvious difference between 1-back and $\mathrm{N}$ back TASK (accuracy: $F(1,29)=165.363, p<0.001, B F_{10}>10^{29}$; RT: $F(1,29)=83.510, p<0.001$, $B F_{10}>10^{16}$ ), an absence of main SESSION effect (accuracy: $F(1,29)=2.475, p=0.127, B F_{10}=0.374$, RT: $\left.F(1,29)=1.124, p=0.298, B F_{10}=0.354\right)$ or interaction between the TASK and the SESSION effect (see Figure 2B; accuracy: $F(1,29)=2.651, p=0.114, B F_{10}=0.550 ; R T: F(1,29)=0.167$, $\left.\mathrm{p}=0.686, \mathrm{BF}_{10}=0.266\right)$.

\section{$\underline{\text { Changes in performance correlate with changes in task avoidance }}$}

We investigated the link between the fatigue-related changes in task avoidance and the other effort and fatigue variables. We found strong evidence for a correlation between fatigue-induced changes in Model Weight and performance in the N-back task (see Figure 4A; Tau=-0.421, $p<0.001, B F_{10}=39.4$ ) but not with 1-back performance (see Figure 4A; Tau=-0.108, $p=0.415$, 
$\left.\mathrm{BF}_{10}=0.33\right)$. This correlation showed that participants exhibited larger effort-discounting when they had decreased performance in the fatigue session. In contrast, Model Weight failed to correlate with MFI (see Figure 4D; Tau $=-0.005, \mathrm{p}=0.972, \mathrm{BF}_{10}=0.235$ ) or NASA scores (see Figure 4B; effort: Tau $=0.122, \mathrm{p}=0.357, \mathrm{BF}_{10}=0.362$; performance: Tau $=0.218, \mathrm{p}=0.094$, $\left.\mathrm{BF}_{10}=0.938\right)$ changes.

Given the correlation between fatigue-related changes in task avoidance and N-back performance, we also looked at the correlation between changes in subjective effort and $\mathrm{N}$-back performance. None of the correlations were significant (neither 1-back nor $\mathrm{N}$-back task performance correlated with NASA performance or effort scores; see Figure $4 \mathrm{C} ; 0.2<\mathrm{BF}_{10}<0.3$ ).

\section{Subjective fatigue correlates with changes in subjective effort and sleepiness}

We also looked at the relation between changes in subjective fatigue and the effort variables. There was a moderate correlation between NASA Effort and MFI (Tau=0.306, $p=0.018$, $\mathrm{BF}_{10}=3.542$ ) and between NASA Effort and $\mathrm{KSS}$ (Tau=0.341, $\mathrm{p}=0.011, \mathrm{BF}_{10}=6.798$ ). Finally, as classically reported in the literature, there was no correlation between the change in MFI and the change in performance (1-back: Tau $=-0.133, p=0.323, B_{10}=0.392 ; N$-back: Tau $=-0.070$, $\mathrm{p}=0.592, \mathrm{BF}_{10}=0.271$ ).

\subsection{Discussion}

In experiment 1, we found only a moderate effect of the CSWm task on the subjective fatigue scores. However, the MFI questionnaire was provided at the end of the whole experiment and therefore, we suspected that the results were affected strongly by the performance of the intervening $\mathrm{N}$-back task. In addition, MFI correlated strongly with KSS, suggesting that the participants failed to distinguish the concept of fatigue from that of sleepiness.

Subjective effort, as assessed through the NASA-TLX questionnaire, increased following the CSWm task, and its change correlated with that of MFI, apparently confirming our hypothesis that MF increases subjective effort cost. However, our other estimate of effort, task avoidance, correlated with behavioral (N-back performance), rather than subjective fatigue (MFI). One issue with the task avoidance estimate was that many participants always selected the difficult task, providing us with no information about their actual estimation of behavioral effort cost. This was presumably caused by their excessive motivation for the monetary reward.

To address these multiple methodological issues, we ran a second experiment to replicate the 
findings in a more refined protocol. One supplementary MFI assessment was added right after the CSWm and documentary-watching sessions. The confound between sleepiness and fatigue was addressed by explaining in detail to each participant the difference between the 2 concepts (Borragán et al., 2017). In addition, in order to restore vigilance, we added an "arousal boost" after the CSWm and documentary-watching sessions and before the final evaluation (to equalize the conditions of questionnaire assessment). Also, we measured arousal by means of pupillometry. The assessment of task avoidance was improved by using virtual coins, rather than directly euros as rewards for the participants. These coins were translated into actual money at the end of the experiment but the participants were not aware of the conversion ratio. Moreover, instead of using all the combinations of difficulty and reward, we used an adaptive procedure to choose the conditions in each trial. Finally, the selection of N-back task difficulty during the training session was also less constrained, with no maximal value for the ' $N$ ' of the $N$ back.

\section{Experiment 2}

\subsection{Materials and methods}

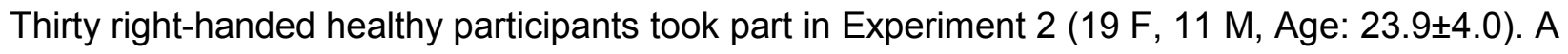
larger proportion of male participants were sought after since males are known to consistently take more financial risks (Charness and Gneezy, 2012). MFI and KSS were assessed at the beginning and at the end of both experimental days, but also between the fatigue/control manipulation and behavioral effort-discounting task, following an arousal boost which consisted in running on the spot for 30 seconds. The arousal boost was introduced to minimize sleepiness. Since it was not expected to have any effect on fatigue, we expected this manipulation to make the MFI a purer measure of fatigue, dissociated from the confound of sleepiness. Each participant read the NASA-TLX instructions carefully prior to the experiment, in order to ensure their appropriate understanding of the different subscales.

The first phase of the training session in Experiment 2 was similar to that of Experiment 1 and comprised 10 blocks, now with 80 letters each. Participants were then engaged in 6 blocks of $\mathrm{N}$ back task with mixed difficulties, similar to those experienced during the following experimental sessions. Different ratios of 1 back \& N-back tasks were performed, while the difficulty level of the $\mathrm{N}$-back (i.e. the value of the $\mathrm{N}$ ) was adapted as a function of participants' individual ability (see Figure 2C). When the participants' performance was greater than or equal to $75 \%$ (mean of 
the $\%$ of targets detected and the $\%$ of distractors correctly rejected) they progressed to the next level. If their performance was below $60 \%$, they reverted back to the inferior $\mathrm{N}$ value. Otherwise they kept practicing the same level. We did not impose maximum difficulty level for the n-back. One hundred and twenty letters were presented in these blocks. Only $3 \mathrm{DL}$ were now evaluated ( $\%$ of N-back: DL-1 $=20 \%, D L-2=50 \%, D L-3=80 \%$ ). The monetary incentive was switched from actual money to a point-based system, and the conversion rate was not disclosed in advance to the participants.

At the beginning of each experimental session, participants performed one warm-up block (DL3). They then had to respond to 40 forced choices, out of which 10 randomly selected blocks were actually performed.

Instead of an exhaustive screening of all possible pairings between difficulty and reward, an adaptive procedure was implemented (Kontsevich and Tyler, 1999; Watson and Pelli, 1983). The idea behind this procedure is to evaluate the expected gain in information (i.e. decrease in entropy) for each possible condition. The algorithm then chooses the reward/difficulty condition that maximizes this expected information gain. Participants' choices were modeled with the same 2-parameter effort-based decision making model as described in Experiment 1. The prior probability distribution $\mathrm{P}_{0}$ of the weight and temperature parameters were set to a normal distribution with mean zero and standard deviation equal to 5 . In each trial, this probability distribution was updated as a function of participant's choice:

$$
\begin{gathered}
\begin{aligned}
& \log (P(\text { weight, temperature } \mid \text { choice, } R, D)) \\
&=\log (P(\text { choice } \mid \text { weight, temperature }, R, D))+\log \left(P_{0}(\text { weight, temperature })\right) \\
&-\log \left(\sum_{w} \sum_{t} P(\text { choice } \mid w, t, R, E) \times P_{0}(w, t)\right)
\end{aligned} \\
P(\text { choice } \mid \text { weight, temperature, } R, D)=\left\{\begin{array}{c}
P(\text { easy } \mid \text { weight }, \text { temperature, } R, D), \text { if easy option chosen } \\
1-P(\text { easy } \mid \text { weight }, \text { temperature }, R, D), \text { if difficult option chosen }
\end{array}\right.
\end{gathered}
$$

Then the expected amount of information gained in the next trial was computed for each condition, each weight and temperature values and each possible choice, and allowed us to extract a probability of choosing a given reward and difficulty condition, $P(R, D)$, according to the following equations:

$$
\begin{gathered}
\log (P(\text { weight, temperature, choice } \mid R, D)) \\
=\log (P(\text { choice } \mid \text { weight, temperature, } R, D))+\log \left(P_{0}(\text { weight, temperature })\right) \\
P(\text { choice } \mid R, D)=\sum_{w} \sum_{t} P(w, t, \text { choice } \mid R, D)
\end{gathered}
$$




$$
\begin{gathered}
\log (P(\text { weight, temperature } \mid \text { choice }, R, D)) \\
=\log (P(\text { weight, temperature, choice } \mid R, D))-\log \left(\sum_{w} \sum_{t} P(w, t, \text { choice } \mid R, D)\right) \\
H(\text { choice }, R, D)=-\sum_{w} \sum_{t} \mathrm{P}(w, t \mid \text { choice, } R, D) \times \log (P(w, t \mid \text { choice }, R, D)) \\
H(R, D)=P(\text { easy } \mid R, D) \times H(\text { easy, } R, D)+P(\text { difficult } \mid R, D) \times H(\text { difficult }, R, D) \\
P(R, D)=\frac{e^{-100 \times(H(R, D-\min (H(R, D)))}}{\sum_{r} \sum_{d} e^{-100 \times(H(r, d)-\min (H(r, d)))}}
\end{gathered}
$$

All other parameters and procedures were identical to Experiment 1.

In order to monitor participant's level of arousal, we recorded pupil size during performance of the N-back task. Pupil size was acquired using an Eyelink 1000+ eye tracker video-based system (SR Research Ltd., Kanata, Ontario, Canada), with a sampling frequency of $500 \mathrm{~Hz}$. We also evaluated maximal pupil dilation by asking the subjects to apply physical force (maximal voluntary contraction, MVC) through a handgrip on a dynamometer Jamar ${ }^{\circledR}$ Hydraulic Hand Dynamometer). This step was performed twice at the beginning and at the end of both experimental days. No pupillometry data were acquired on the training day.

\subsection{Results}

\section{CSWm task showed time-on-task increase in conflict and switch cost}

In Experiment 2, performance in the CSWm task was $93 \pm 1 \%, 95 \pm 1 \%, 44 \pm 2 \%$ and $84 \pm 2 \%$ (mean $\pm 95 \%$ confidence interval) for congruent/switch, congruent/no-switch, incongruent/switch and incongruent/no-switch trials, respectively. Similarly to Experiment 1, the incongruency effect increased over time in the switch $\left(\mathrm{BF}_{10}>10^{6}\right)$, but decreased in no-switch trials $\left(\mathrm{BF}_{10}=8.33\right)$, while the effect of switch on accuracy increased over time in the incongruent $\left(\mathrm{BF}_{10}>60000\right)$ but decreased in the congruent trials $\left(\mathrm{BF}_{10}=924\right)$. Again, correlation of these correlation coefficients with the other subjective and behavioral measures (NASA scores, N-back performance scores, model parameters, MFI, IMI and KSS) resulted in no significant outcome $\left(\mathrm{BF}_{10}<3\right)$.

\section{$\underline{\text { CSWm session increases MFI, decreases performance but leaves task avoidance unchanged }}$}

We investigated the effect of the fatigue-inducing task on the dependent variables. A repeatedmeasure ANOVA on the post-pre difference in MFI and KSS scores showed a strong effect of SESSION on KSS (see Figure 3D; $F(1,29)=10.69, p=0.003, B_{10}=15.32$ ) and a decisive effect on the $\operatorname{MFI}\left(F(1,29)=23.06, p<0.001, B F_{10}=408.2\right)$. There was a moderate correlation between the fatigue-control changes in the two variables (Kendall's tau=0.303, $p=0.026, \quad B F_{10}=3.377$ ), 
showing that the changes brought to Experiment 2 were only partly effective in separating fatigue and sleepiness (reduction of the correlation coefficient from 0.5 to 0.3 ). Similarly to Experiment 1, there was neither SESSION effect on $\operatorname{IMI}\left(F(1,29)=1.965, p<0.172, B_{10}=0.657\right)$, nor correlation between IMI and MFI (Kendall's tau=0.016, $\mathrm{p}=0.900, \mathrm{BF}_{10}=0.237$ ) or IMI and KSS (Kendall's tau $=-0.015, p=0.914, B_{10}=0.237$ ).

Regarding the NASA-TLX variables, we confirmed that the NASA effort and performance scores changed as a function of the DIFFICULTY level (NASA effort: $F(2,58)=26.654, p<0.001$, $B F_{\text {inclusion }}=2.880 \times 10^{7}, N A S A$ performance: $F(2,58)=14.884, p<0.001, B F_{\text {inclusion }}=10126$ ) but not SESSION (see Figure 3F; NASA effort: $F(1,29)=0.294, p=0.592, \quad B F_{\text {inclusion }}=0.203$, NASA performance: $F(1,29)=0.124, p=0.728, B F_{\text {inclusion }}=0.171$ ) or SESSION $\times$ DIFFICULTY interaction (NASA effort: $F(2,58)=0.929, p=0.401, B F_{\text {inclusion }}=0.166$, NASA performance: $F(2,58)=0.257$, $\mathrm{p}=0.775, \mathrm{BF}_{\text {inclusion }}=0.116$ ). This time, there was a moderate, negative correlation between the fatigue-related changes in the two variables (Kendall's tau $=-0.343, \mathrm{p}=0.007, \mathrm{BF}_{10}=7.037$ ).

We then looked at the task avoidance variables. The temperature and weight parameters from the task avoidance models were also subjected to a one-way Bayesian repeated-measure ANOVA with SESSION (control vs fatigue) as factor. There was no change in either parameters (see Figure $2 \mathrm{~A}$, right panel and Figure $3 \mathrm{E}$; temperature: $\mathrm{F}(1,29)=1.868, \mathrm{p}=0.182, \mathrm{BF}_{10}=0.594$, weight: $\left.F(1,29)=0.001, p=0.981, B_{10}=0.263\right)$ between sessions. There was no correlation between the fatigue-control changes in the two variables (Kendall's tau $=0.222, p=0.092$, $\mathrm{BF}_{10}=0.978$ ). The percentage of acceptance of the difficult option in Experiment 2 was $61.0 \pm 4.2 \%$ in the control and $58.4 \pm 5.4 \%$ in the fatigue session (mean $\pm \mathrm{Cl}$ ). None of the participants picked systematically the difficult option (all acceptance percentages were below $90 \%)$.

In Experiment 2, we found that fatigue had a significant impact on $\mathrm{N}$-back task performance (see figure 2B). A RM-ANOVA on the logit-transformed accuracy scores showed, beside the difference between 1-back and N-back TASK (accuracy: $F(1,29)=299.4, p<0.001, B F_{10}>10^{37}, R T$ : $\left.F(1,29)=56.587, p<0.001, B F_{10}>10^{15}\right)$, a moderate interaction between the TASK and the SESSION effect on accuracy $\left(F(1,29)=7.789, p=0.009, B F_{10}=4.979\right)$. This interaction was absent from the $R T$ data $\left(F(1,29)=0.105, p=0.748, B_{10}=0.255\right)$. The main SESSION effect was only anecdotal (accuracy: $\mathrm{F}(1,29)=11.354, \mathrm{p}=0.002, \mathrm{BF}_{10}=2.593, \mathrm{RT}: \mathrm{F}(1,29)=8.024, \mathrm{p}=0.008, \mathrm{BF}_{10}=1.159$ ). Looking at the accuracy in each task individually, we found a strong decrease in performance with fatigue in the 1-back task $\left(F(1,29)=10.34, p=0.003, B_{10}=12.97\right)$ but not in the $\mathrm{N}$-back task $\left(F(1,29)=0.132, p=0.719, B_{10}=0.276\right)$. There was no correlation between the fatigue-control 
changes in the two variables (Kendall's tau=0.145, $p=0.272, B F_{10}=0.432$ ).

Decrease in performance correlates with changes in task avoidance and subjective effort

In agreement with Experiment 1, we found strong evidence for a correlation between fatigueinduced changes in Model Weight and performance (see Figure 4E; N-back: Tau=-0.374, $\mathrm{p}=0.004, \mathrm{BF}_{10}=13.376$; 1-back: Tau=-0.374, $\mathrm{p}=0.004, \mathrm{BF}_{10}=13.376$ ). In contrast, Model Weight changes failed to correlate with MFI (see Figure $4 \mathrm{H}$; Tau=-0.082, $\mathrm{p}=0.531, \mathrm{BF}_{10}=0.286$ ), KSS (Tau=-0.223, $p=0.110, B_{10}=0.999$ ) or NASA score changes (see Figure 4F; effort: Tau=0.109, $\mathrm{p}=0.401, \mathrm{BF}_{10}=0.332$; performance: $\mathrm{Tau}=-0.095, \mathrm{p}=0.464, \mathrm{BF}_{10}=0.306$ ).

There was also a positive correlation between fatigue-induced changes in performance in the $\mathrm{N}$ back task and NASA performance score (see Figure 4G, blue; N-back: Tau=0.393, $p=0.002$, $\mathrm{BF}_{10}=20.615$; 1-back performance: Tau=0.264, $\mathrm{p}=0.041, \mathrm{BF}_{10}=1.784$ ), and a moderate correlation of the performance changes with the NASA effort score (see Figure 4G, red; N-back: Tau=-0.306, $p=0.018, B F_{10}=3.531 ; 1$-back: Tau=-0.149, $\left.p=0.256, B F_{10}=0.450\right)$.

No relation between fatigue and arousal

In Experiment 2, we measured pupil size in order to disentangle cognitive fatigue from unspecific time-on-task effects on arousal. Baseline arousal levels remained unaffected by the fatigue manipulation, as indexed by average pupil size during the MVC task $(F(1,29)=0.250$, $\mathrm{p}=0.621, \mathrm{BF}_{10}=0.281$ ). The change in pupil-indexed arousal correlated moderately with the change in KSS score but not with the MFI score change (KSS: Tau=0.302, $p=0.025$, $\mathrm{BF}_{10}=3.294 ; \mathrm{MFI}$ : Tau=-0.005, $\left.\mathrm{p}=0.971, \mathrm{BF}_{10}=0.235\right)$.

A 2-way Bayesian RM-ANOVA on the peak pupil response during the task showed a moderate effect of session $\left(F(1,29)=1.912, p=0.177, B F_{\text {inclusion }}=3.157\right)$, with larger pupil responses in the fatigue session. There was no DIFFICULTY $\left(F(2,58)=1.573, p=0.216, \quad B F_{\text {inclusion }}=0.092\right)$ or interaction $\left(F(2,58)=2.662, p=0.078, B F_{\text {inclusion }}=0.245\right)$ effect. The between-session change in pupil size failed to correlate with any of the fatigue measures $\left(\mathrm{BF}_{\text {inclusion }}\right.$ between 0.2 and 0.3$)$.

Subjective fatigue failed to correlate with the other fatigue or effort measures

Beside the previous correlations reported above, there was no correlation between NASA Effort and MFI (Tau=-0.012, p=0.929, $\mathrm{BF}_{10}=0.236$ ) or $\mathrm{KSS}$ (Tau=-0.090, $\left.\mathrm{p}=0.504, \mathrm{BF}_{10}=0.238\right)$. Finally, there was no correlation between the change in MFI and the change in performance (1-back: Tau=-0.100, $p=0.442, B_{10}=0.357 ; \mathrm{N}$-back: Tau=0.063, $\mathrm{p}=0.629, \mathrm{BF}_{10}=0.264$ ). 


\subsection{Discussion}

Important methodological limitations from Experiment 1 were addressed in the second experiment. After observing that behavioral effort cost was not affected by fatigue, and that many participants never selected the easy task, we hypothesized that the N-back task may not have been challenging enough and that the value of reward was too high relative to effort cost. Therefore, in Experiment 2 we did not restrict the difficulty of the working memory task, such that some participants were able to perform a 9-back version of the task. In order to decrease the incentive value of reward, we switched from using actual money to a point-based system. Finally, instead of testing exhaustively every possible combination of task difficulty and reward, we implemented a Bayesian search procedure, in which the proposed options were adapted based on the previous responses. Despite these improvements, one remaining limitation is noteworthy. We tested young university students, who are generally strongly motivated by reward and this may have masked partly the increases in behavioral effort costs associated with fatigue.

In experiment 2, we found a decisive effect of the CSWm task on the subjective fatigue scores, evaluated immediately following the fatigue-inducing manipulation, and on N-back performance. In contrast to Experiment 1 , MFI correlated only moderately with KSS, confirming that better explanation of the fatigue and sleepiness constructs to the participants and/or the arousal boost manipulation decreased the confounding effect of sleepiness on fatigue. Average tonic pupil size, used as an independent measure of arousal, showed no systematic change across sessions, but correlated with the subjective sleepiness measure. Interestingly, however, we found that peak pupillary responses, thought to be indicative of the phasic variations in noradrenaline and/or acetylcholine (Aston-Jones and Cohen, 2005; Joshi et al., 2016; Reimer et al., 2016), increased following the fatigue manipulation, in agreement with earlier studies (Wright et al., 2007).

Another difference with respect to Experiment 1 was that the fatigue manipulation failed to have any impact on the subjective effort perception measures. We can only speculate on the reason for this difference, but one may suggest that subjective effort increased in Experiment 1 following the CSWm task because of increased sleepiness, rather than because of cognitive fatigue. In Experiment 2, since the impact of sleepiness was decreased, subjective effort did not change significantly.

Finally, the most important outcome of Experiment 2 is that we were able to replicate the two main findings from Experiment 1: task avoidance correlated with fatigue-induced performance 
decrement but not with subjective fatigue.

\section{General discussion}

In the present paper, we hypothesized that cognitive fatigue would increase the cost of cognitive effort. We evaluated fatigue and effort in terms of subjective feeling, as well as in terms of behavioral manifestation such as task performance and reward discounting, respectively. We found that extensive involvement in a cognitively demanding task increased the feeling of fatigue and impaired task performance in the easy version of the task. Throughout the experiment, preference of the participants between task options reflected the attribution of a cost to cognitive effort (Westbrook et al., 2013). However, contrary to the hypothesis, effort cost did not increase with subjective fatigue but increased rather in proportion to fatigue-induced decrease in performance.

The cognitive mechanisms and neural substrates of effort and fatigue remain poorly understood. If subjective fatigue was a protective mechanism against forthcoming resource depletion (Gergelyfi et al., 2015; Muraven and Slessareva, 2003), or if it was the manifestation of increased engagement to maintain performance despite resource disruption (Hockey, 1997), it should also have increased task avoidance. The present findings go clearly against this hypothesis, by showing an absence of link between subjective fatigue and task avoidance. It is worth mentioning that we found a positive correlation between subjective fatigue and subjective effort in Experiment 1. However, this correlation was weak and disappeared in Experiment 2, in which we addressed multiple methodological limitations. In contrast, task avoidance correlated in both experiments with fatigue-related performance decrement. These findings emphasize the dissociation between the subjective and behavioral manifestations of fatigue and effort. Indeed, in both experiments, subjective and behavioral assessments of fatigue and effort failed to correlate with each other.

Parts of the present findings rest on the exclusion of the alternative hypothesis. Bayesian statistics, by considering that probabilities can be attributed to hypotheses (e.g. there is a $10 \%$ chance of raining tomorrow), allow us to evaluate the differences in likelihood between the null and alternative hypotheses in terms of Bayes Factor. Here, the correlation between MFI and model weight, reflecting task avoidance, resulted in both experiments in substantial evidence for the null hypothesis (Bayes Factor <0.3). This replication of the null result provides, to our view, strong evidence that MFI, as a general measure of subjective fatigue, is not associated with increased cognitive task avoidance. However, it is important to note that this finding does not demonstrate that other phenomenological aspects of fatigue, that would fail to be captured by 
the MFI, could not lead to increased task avoidance. Along the same line, it is possible that longer task performance, or more demanding tasks, could have led to increased task avoidance. Nevertheless, 2 hours of a task known to tax cognitive control resources, and in which difficulty was adjusted to lead to performance well below maximum, were not enough to induce increased task avoidance in the present experiment (BF below 0.3 in Exp2 and slightly above in Exp1).

We found that despite successful fatigue inducement, the participants maintained similar performance in the $\mathrm{N}$-back task but showed significant decrease in 1-back performance. Our initial hypothesis was that fatigue was associated with disruption of vigilance, which typically leads to more mistakes in easy and monotonous tasks (Thiffault and Bergeron, 2003). We accounted for this possibility in Experiment 2, by adding an arousal boost manipulation to restore vigilance, by improving the dissociation between fatigue and sleepiness self-evaluation in participants, and by measuring participants' pupil size (Sara and Bouret, 2012; Zénon et al., 2014). We found neither change of arousal between the sessions nor any correlation with the measures of fatigue, sleepiness and performance. This suggests that the decrement in 1-back performance was not caused by decreased vigilance, even though measuring vigilance directly with psychomotor vigilance task would be necessary to confirm this claim more decisively. Therefore, understanding why fatigue disrupts specifically 1-back performance will require further investigation.

One potential issue with the neuroeconomical evaluation procedure is that the devaluation of reward value associated with task difficulty may be influenced by performance in the task, and not only by effort. This issue deserves a careful consideration in the present study, given that we found a correlation between fatigue-induced changes in task avoidance and performance. Previous attempts at solving this issue were based on persuading participants that their reward depended on task engagement, rather than on performance (Westbrook et al., 2013). Here, we relied on the design of the task-choice procedure to avoid any influence of performance on participants' decisions. Since block performance was computed by averaging accuracy in both the difficult and the easy tasks irrespective of their proportion in the block, a poor performance in the difficult task would have the same consequence on the reward, whatever choice the participants made. Therefore, there was no incentive for the participants to change their preference for the easy task ratio following fatigue. In fact, if participants taskchoice depended on auto-evaluation of performance, decreased performance in N-back would have increased preference for low DL task conditions, whereas decreased performance in 1- 
back would have increased preference for large DL tasks. However, we found that performance in both the 1-back and N-back tasks correlated with task avoidance. This indicates that task avoidance was affected by fatigue-induced performance decline rather than by the integration of performance as a cost in participants' decision. One question to address in future studies is whether the presence of the performance feedback at the end of each block is necessary to induce this relationship between performance decrements and task avoidance.

Another possible limitation of the present study is the reliance on the CSWm task to induce fatigue and N-back tasks to measure its effect. This choice was justified on the basis of several earlier studies of fatigue and effort discounting (Moeller et al., 2012; Pageaux et al., 2015; Wang et al., 2016; Westbrook et al., 2013). In addition, since we were interested in the link between the subjective feeling of fatigue - a task-independent subjective percept - and cognitive effort, there was no need to use the same task to induce and measure fatigue. Using different tasks has also the advantage of limiting the problem of boredom associated with the performance of the same monotonous task for several hours (Bench and Lench, 2013). Since little is known regarding the transferability of fatigue between different cognitive processes - available evidence point sometimes in the direction of global effect of fatigue, independent of the processes involved in fatigue induction (Arai, 1912; Wright et al., 2007), sometimes in the opposite direction (Persson et al., 2007) -, we decided to use a fatigue-inducing task involving a large range of cognitive processes, among which some should overlap with the ones involved in $\mathrm{N}$-back performance. Along the same line, it could be argued that the N-back task was not demanding enough to lead to measurable effects on behavioral effort cost. We think it is unlikely because hit rate was only $80 \%$ on average in Experiment 2 and we found clear effect of task difficulty on reward discounting. Finally, the potential influence of the affective state of the participants (positive or negative) was not specifically evaluated following the completion of the fatigue / control manipulation (except for intrinsic motivation, which remained unaffected by fatigue induction). Affective state can indeed influence performance in cognitive tasks (Carver, C. S., \& Scheier, 2011; Vohs et al., 2008) and should be considered as dependent variable in future studies.

It has been shown frequently that the feeling of fatigue occurs before any decrease of performance (Mullette-Gillman et al., 2015). Compensatory control hypothesis states that during the initial phase of cognitive fatigue, performance can be maintained at the cost of higher subjective effort (Hockey, 2013, 1997). However, the results of the present study failed to corroborate this hypothesis by showing a clear lack of relationship between subjective fatigue 
and effort. We suggest that this lack of correlation of subjective fatigue with both performance decrement and effort is compatible with an alternative "anticipatory regulation" view, according to which cognitive fatigue would be an anticipatory protection mechanism, akin to anticipatory regulatory mechanisms described in the physical fatigue literature (Crewe et al., 2008; Tucker, 2009; Tucker and Noakes, 2009). This protection mechanism would anticipate future adverse consequences associated with task execution and would urge subjects to stop before any actual consequence occurs (van der Linden, 2011). In other words, while the compensatory hypothesis assumes that fatigue would affect cognitive resources early on and would lead to compensatory increase in the recruitment of further resources in order to maintain performance (Hockey, 2011), the anticipatory regulation hypothesis assumes that subjective fatigue occurs before any adverse consequence can be observed on the brain. According to this view, the engagement of resources would remain constant during the initial buildup of fatigue, which would thus not lead necessarily to increased subjective perception of effort or increased effort-discounting. Actual alteration of cognitive resources would occur if one continues to engage in the task despite subjective fatigue. At that point, performance would start to decrease and effortful control would be engaged to compensate that decrease. This, in turn, might explain our findings that task avoidance correlates with performance decrement.

This putative reliance of fatigue on such anticipatory mechanism points to several candidates in terms of potential neural substrates. Anterior insula and cingulate cortex, in particular, have been shown to encode anticipatory interoceptive signals (Barrett and Simmons, 2015). Interestingly, these same regions are also thought to be involved in the phenomenology of fatigue (Boksem and Tops, 2008; Dantzer et al., 2014; Pavese et al., 2010). Naturally, the present findings provide only indirect support for this anticipatory regulation view and further computational and neuroimaging studies are required to assess its validity in depth.

\section{Acknowledgements}

This work was supported by Fonds de la Recherche Scientifique (FNRS-FDP), Fondation Médicale Reine Elisabeth (FMRE), the Fondation Louvain and IdEx Bordeaux. The authors would like to acknowledge the contribution of Simon Van Hemelrijck for his support in data acquisition. 


\section{References}

Ackerman, P.L., Kanfer, R., 2009. Test length and cognitive fatigue: An empirical examination of effects on performance and test-taker reactions. J. Exp. Psychol. Appl. 15, 163-181. doi:10.1037/a0015719

Åkerstedt, T., Anund, A., Axelsson, J., Kecklund, G., 2014. Subjective sleepiness is a sensitive indicator of insufficient sleep and impaired waking function. J. Sleep Res. 23, 240-252. doi:10.1111/jsr.12158

Åkerstedt, T., Gillberg, M., 1990. Subjective and Objective Sleepiness in the Active Individual. Int. J. Neurosci. 52, 29-37. doi:10.3109/00207459008994241

Arai, T., 1912. Mental fatigue. Unpublished doctoral dissertation, Columbia University.

Aston-Jones, G., Cohen, J.D., 2005. An integrative theory of locus coeruleus-norepinephrine function: adaptive gain and optimal performance. Annu Rev Neurosci.

Badin, O.O., Smith, M.R., Conte, D., Coutts, A.J., 2016. Mental fatigue: Impairment of technical performance in small-sided soccer games. Int. J. Sports Physiol. Perform. 11, 1100-1105. doi:10.1123/ijspp.2015-0710

Bailey, A., Channon, S., Beaumont, J.G., 2007. The relationship between subjective fatigue and cognitive fatigue in advanced multiple sclerosis. Mult. Scler. J. 13, 73-80. doi:10.1177/1352458506071162

Barrett, L.F., Simmons, W.K., 2015. Interoceptive predictions in the brain. Nat. Rev. Neurosci. doi: $10.1038 / \mathrm{nrn} 3950$

Bench, S., Lench, H., 2013. On the Function of Boredom. Behav. Sci. (Basel). 3, 459-472. doi:10.3390/bs3030459

Boksem, M.A.S., Meijman, T.F., Lorist, M.M., 2006. Mental fatigue, motivation and action monitoring. Biol. Psychol. 72, 123-132.

Boksem, M.A.S., Tops, M., 2008. Mental fatigue: Costs and benefits. Brain Res. Rev. doi:10.1016/j.brainresrev.2008.07.001

Borragán, G., Slama, H., Bartolomei, M., Peigneux, P., 2017. Cognitive fatigue: A Time-based Resource-sharing account. Cortex 89, 71-84. doi:10.1016/j.cortex.2017.01.023

Brainard, D.H., 1997. The Psychophysics Toolbox. Spat. Vis. 10, 433-436. doi:10.1163/156856897X00357 
Campagne, A., Pebayle, T., Muzet, A., 2004. Correlation between driving errors and vigilance level: Influence of the driver's age. Physiol. Behav. 80, 515-524.

doi:10.1016/j.physbeh.2003.10.004

Carter, E.C., Kofler, L.M., Forster, D.E., McCullough, M.E., 2015. A series of meta-analytic tests of the depletion effect: Self-control does not seem to rely on a limited resource. J. Exp. Psychol. Gen. 144, 796-815. doi:10.1037/xge0000083

Carver, C. S., \& Scheier, M.F., 2011. Self-Regulation of Action and Affect, in: Baumeister, K.D.V.\& R.F. (Ed.), Handbook of Self Regulation: Research, Theory and Applications. Guilford Press., New York, NY, USA, pp. 3-21.

Charness, G., Gneezy, U., 2012. Strong Evidence for Gender Differences in Risk Taking. J. Econ. Behav. Organ. 83, 50-58. doi:10.1016/j.jebo.2011.06.007

Christie, S.T., Schrater, P., 2015. Cognitive cost as dynamic allocation of energetic resources. Front. Neurosci. 9, 1-15. doi:10.3389/fnins.2015.00289

Crewe, H., Tucker, R., Noakes, T.D., 2008. The rate of increase in rating of perceived exertion predicts the duration of exercise to fatigue at a fixed power output in different environmental conditions. Eur. J. Appl. Physiol. 103, 569-577. doi:10.1007/s00421-0080741-7

Dantzer, R., Heijnen, C.J., Kavelaars, A., Laye, S., Capuron, L., 2014. The neuroimmune basis of fatigue. Trends Neurosci. doi:10.1016/j.tins.2013.10.003

Deluca, J., 2005. Fatigue, cognition and mental effort, in: Fatigue as a Window to the Brain. p. $17: 52$.

Dorrian, J., Roach, G.D., Fletcher, A., Dawson, D., 2007. Simulated train driving: Fatigue, selfawareness and cognitive disengagement. Appl. Ergon. 38, 155-166. doi:10.1016/j.apergo.2006.03.006

Fairclough, S.H., Houston, K., 2004. A metabolic measure of mental effort. Biol. Psychol. 66, 177-190. doi:10.1016/j.biopsycho.2003.10.001

Gailliot, M.T., Baumeister, R.F., 2007. The physiology of willpower: Linking blood glucose to self-control. Personal. Soc. Psychol. Rev. 11, 303-327. doi:10.1177/1088868307303030

Gelman, A., Tuerlinckx, F., 2000. Type S error rates for classical and Bayesian single and multiple comparison procedures. Comput. Stat. 15, 373-390. doi:10.1007/s001800000040 
Gentile, S., Delarozière, J.C., Favre, F., Sambuc, R., San Marco, J.L., 2003. Validation of the French "multidimensional fatigue inventory" (MFI 20). Eur. J. Cancer Care (Engl). 12, 5864. doi:10.1046/j.1365-2354.2003.00295.x

Gergelyfi, M., Jacob, B., Olivier, E., Zénon, A., 2015. Dissociation between mental fatigue and motivational state during prolonged mental activity. Front. Behav. Neurosci. 9. doi:10.3389/fnbeh.2015.00176

Hart, S.G., Staveland, L.E., 1988. Development of NASA-TLX (Task Load Index): Results of Empirical and Theoretical Research. Adv. Psychol. 52, 139-183. doi:10.1016/S01664115(08)62386-9

Hockey, G.R., 1997. Compensatory control in the regulation of human performance under stress and high workload; a cognitive-energetical framework. Biol. Psychol. 45, 73-93.

Hockey, G.R.J., 2011. A Motivational Control Theory of Cognitive Fatigue. Cogn. fatigue Multidiscip. Perspect. Curr. Res. Futur. Appl. 167-187. doi:10.1037/12343-008

Holtzer, R., Shuman, M., Mahoney, J.R., Lipton, R., Verghese, J., 2011. Cognitive Fatigue defined in the context of attention networks. Aging, Neuropsychol. Cogn. 18, 108-128. doi:10.1080/13825585.2010.517826

Hosking, J.G., Cocker, P.J., Winstanley, C.A., 2014. Dissociable contributions of anterior cingulate cortex and basolateral amygdala on a rodent cost/benefit decision-making task of cognitive effort. Neuropsychopharmacology 39, 1558-67. doi:10.1038/npp.2014.27

Inzlicht, M., Bartholow, B.D., Hirsh, J.B., 2015. Emotional foundations of cognitive control. Trends Cogn. Sci. doi:10.1016/j.tics.2015.01.004

Joshi, S., Li, Y., Kalwani, R.M., Gold, J.I., 2016. Relationships between Pupil Diameter and Neuronal Activity in the Locus Coeruleus, Colliculi, and Cingulate Cortex. Neuron 89, 221234. doi:10.1016/j.neuron.2015.11.028

Kass, R.E., Raftery, A.E., 1995. Bayes Factor. J. Am. Stat. Assoc. doi:10.2307/2291091

Kato, Y., Endo, H., Kizuka, T., 2009. Mental fatigue and impaired response processes: Eventrelated brain potentials in a Go/NoGo task. Int. J. Psychophysiol. 72, 204-211. doi:10.1016/j.ijpsycho.2008.12.008

Kirchner, W.K., 1958. Age differences in short-term retention of rapidly changing information. J. Exp. Psychol. 55, 352-358. doi:10.1037/h0043688 
Kontsevich, L.L., Tyler, C.W., 1999. Bayesian adaptive estimation of psychometric slope and threshold. Vision Res. 39, 2729-2737. doi:10.1016/S0042-6989(98)00285-5

Krueger, J.M., Rector, D.M., Roy, S., Van Dongen, H.P.A., Belenky, G., Panksepp, J., 2008. Sleep as a fundamental property of neuronal assemblies. Nat. Rev. Neurosci. 9, 910-919. doi:10.1038/nrn2521

Kurzban, R., Duckworth, A., Kable, J.W., Myers, J., 2013. An opportunity cost model of subjective effort and task performance. Behav. Brain Sci. 36, 661-679. doi:10.1017/S0140525X12003196

Lim, J., Wu, W. chau, Wang, J., Detre, J.A., Dinges, D.F., Rao, H., 2010. Imaging brain fatigue from sustained mental workload: An ASL perfusion study of the time-on-task effect. Neuroimage 49, 3426-3435. doi:10.1016/j.neuroimage.2009.11.020

Lorist, M.M., 2008. Impact of top-down control during mental fatigue. Brain Res. 1232, 113-123. doi:10.1016/j.brainres.2008.07.053

Lorist, M.M., Klein, M., Nieuwenhuis, S., De Jong, R., Mulder, G., Meijman, T.F., 2000. Mental fatigue and task control: planning and preparation. Psychophysiology 37, 614-625.

MacLeod, C.M., 1991. Half a century of research on the Stroop effect: an integrative review. Psychol. Bull. 109, 163-203. doi:Doi 10.1037//0033-2909.109.2.163

Massar, S.A.A., Wester, A.E., Volkerts, E.R., Kenemans, J.L., 2010. Manipulation specific effects of mental fatigue: Evidence from novelty processing and simulated driving. Psychophysiology 47, 1119-1126. doi:10.1111/j.1469-8986.2010.01028.x

Moeller, S.J., Tomasi, D., Honorio, J., Volkow, N.D., Goldstein, R.Z., 2012. Dopaminergic involvement during mental fatigue in health and cocaine addiction. Transl. Psychiatry 2 , e176.

Mullette-Gillman, O.A., Leong, R.L.F., Kurnianingsih, Y.A., 2015. Cognitive Fatigue Destabilizes Economic Decision Making Preferences and Strategies. PLoS One 10, e0132022. doi:10.1371/journal.pone.0132022

Muraven, M., Slessareva, E., 2003. Mechanisms of Self-Control Failure: Motivation and Limited Resources. Personal. Soc. Psychol. ....

Pageaux, B., Marcora, S.M., Rozand, V., Lepers, R., 2015. Mental fatigue induced by prolonged self-regulation does not exacerbate central fatigue during subsequent whole-body endurance exercise. Front. Hum. Neurosci. 9. doi:10.3389/fnhum.2015.00067 
Pavese, N., Metta, V., Bose, S.K., Chaudhuri, K.R., Brooks, D.J., 2010. Fatigue in Parkinson 's disease is linked to striatal and limbic serotonergic dysfunction 3434-3443. doi:10.1093/brain/awq268

Persson, J., Welsh, K.M., Jonides, J., Reuter-Lorenz, P.A., 2007. Cognitive fatigue of executive processes: Interaction between interference resolution tasks. Neuropsychologia 45, 15711579. doi:10.1016/j.neuropsychologia.2006.12.007

Reimer, J., Mcginley, M.J., Liu, Y., Rodenkirch, C., Wang, Q., Mccormick, D.A., Tolias, A.S., 2016. Pupil fluctuations track rapid changes in adrenergic and cholinergic activity in cortex. Nat. Publ. Gr. 7, 1-7. doi:10.1038/ncomms13289

Ryan, R.M., Deci, E.L., 2000. Self-determination theory and the facilitation of intrinsic motivation, social development, and well-being. Am. Psychol. 55, 68-78. doi:10.1037/0003-066X.55.1.68

Sara, S.J., Bouret, S., 2012. Orienting and Reorienting: The Locus Coeruleus Mediates Cognition through Arousal. Neuron 76, 130-141. doi:10.1016/j.neuron.2012.09.011

Schwid, S.R., Tyler, C.M., Scheid, E.A., Weinstein, A., Goodman, A.D., McDermott, M.P., 2003. Cognitive fatigue during a test requiring sustained attention: a pilot study. Mult. Scler. J. 9, 503-508. doi:10.1191/1352458503ms946oa

Shenhav, A., Musslick, S., Lieder, F., Kool, W., Griffiths, T.L., Cohen, J.D., Botvinick, M.M., 2017. Toward a Rational and Mechanistic Account of Mental Effort. Annu. Rev. Neurosci. 40, 99-124. doi:10.1146/annurev-neuro-072116-031526

Stewart, C.C., Wright, R.A., Azor Hui, S.K., Simmons, A., 2009. Outcome expectancy as a moderator of mental fatigue influence on cardiovascular response. Psychophysiology 46, 1141-1149. doi:10.1111/j.1469-8986.2009.00862.x

Tanaka, M., 2015. Effects of Mental Fatigue on Brain Activity and Cognitive Performance: A Magnetoencephalography Study. Anat. Physiol. s4. doi:10.4172/2161-0940.S4-002

Thiffault, P., Bergeron, J., 2003. Monotony of road environment and driver fatigue: A simulator study. Accid. Anal. Prev. 35, 381-391. doi:10.1016/S0001-4575(02)00014-3

Tucker, R., 2009. The anticipatory regulation of performance: The physiological basis for pacing strategies and the development of a perception-based model for exercise performance. Br. J. Sports Med. doi:10.1136/bjsm.2008.050799

Tucker, R., Noakes, T.D., 2009. The physiological regulation of pacing strategy during exercise: 
A critical review. Br. J. Sports Med. doi:10.1136/bjsm.2009.057562

Vadillo, M.A., Gold, N., Osman, M., 2016. The Bitter Truth About Sugar and Willpower: The Limited Evidential Value of the Glucose Model of Ego Depletion. Psychol. Sci. 27, 12071214. doi:10.1177/0956797616654911

Van Cutsem, J., De Pauw, K., Buyse, L., Marcora, S., Meeusen, R., Roelands, B., 2017. Effects of mental fatigue on endurance performance in the heat. Med. Sci. Sports Exerc. 49, 16771687. doi:10.1249/MSS.0000000000001263

van der Linden, D., 2011. The urge to stop: The cognitive and biological nature of acute mental fatigue. Cogn. Fatigue Multidiscip. Perspect. Curr. Res. Futur. Appl. 149-164. doi:10.1037/12343-007

van der Linden, D., Frese, M., Meijman, T.F., 2003. Mental fatigue and the control of cognitive processes: effects on perseveration and planning. Acta Psychol. (Amst). 113, 45-65.

Vohs, K.D., Baumeister, R.F., Schmeichel, B.J., Twenge, J.M., Nelson, N.M., Tice, D.M., 2008. Making Choices Impairs Subsequent Self-Control: A Limited-Resource Account of Decision Making, Self-Regulation, and Active Initiative. J. Pers. Soc. Psychol. 94, 883-898. doi:10.1037/0022-3514.94.5.883

Vyazovskiy, V. V., Olcese, U., Hanlon, E.C., Nir, Y., Cirelli, C., Tononi, G., 2011. Local sleep in awake rats. Nature 472, 443-447. doi:10.1038/nature10009

Wang, C., Trongnetrpunya, A., Samuel, I.B.H., Ding, M., Kluger, B.M., 2016. Compensatory Neural Activity in Response to Cognitive Fatigue. J. Neurosci. 36, 3919-24. doi:10.1523/JNEUROSCI.3652-15.2016

Watson, A.B., Pelli, D.G., 1983. Quest: A Bayesian adaptive psychometric method. Percept. Psychophys. 33, 113-120. doi:10.3758/BF03202828

Westbrook, A., Braver, T.S., 2015. Cognitive effort: A neuroeconomic approach. Cogn. Affect. Behav. Neurosci. 15, 395-415. doi:10.3758/s13415-015-0334-y

Westbrook, A., Kester, D., Braver, T.S., 2013. What Is the Subjective Cost of Cognitive Effort? Load, Trait, and Aging Effects Revealed by Economic Preference. PLoS One 8, e68210. doi:10.1371/journal.pone.0068210

Wright, R.A., Junious, T.R., Neal, C., Avello, A., Graham, C., Herrmann, L., Junious, S., Walton, N., 2007. Mental fatigue influence on effort-related cardiovascular response: Difficulty effects and extension across cognitive performance domains. Motiv. Emot. 31, 219-231. 
doi:10.1007/s11031-007-9066-9

Zénon, A., Duclos, Y., Carron, R., Witjas, T., Baunez, C., Régis, J., Azulay, J.P., Brown, P., Eusebio, A., 2016. The human subthalamic nucleus encodes the subjective value of reward and the cost of effort during decision-making. Brain 139, 1830-1843. doi:10.1093/brain/aww075

Zénon, A., Sidibé, M., Olivier, E., 2014. Pupil size variations correlate with physical effort perception. Front. Behav. Neurosci. 8. doi:10.3389/fnbeh.2014.00286

Zering, J.C., Brown, D.M.Y., Graham, J.D., Bray, S.R., 2017. Cognitive control exertion leads to reductions in peak power output and as well as increased perceived exertion on a graded exercise test to exhaustion. J. Sports Sci. 35, 1799-1807.

doi:10.1080/02640414.2016.1237777 


\section{Figure legends}

Figure 1. Schematic description of the protocol. Participants were first evaluated with the multidimensional fatigue inventory (MFI) and Karolinska sleepiness scale (KSS). Following fatigue-inducing or control manipulation they again completed the questionnaires (only intrinsic motivation inventory (IMI) in Exp 1), and performed a forced-choice working memory task followed by the National Aeronautics and Space Administration Task Load Index (NASA-TLX). Experiment 2 also included arousal boosts consisting of 30-second run on the spot, prior to questionnaires completion.

Figure 2. A. Fitting curves from the behavioral model, averaged across participants for each difficulty level and session. Task preferences indicate a clear effect of difficulty level on probability of accepting more difficult task, with no clear difference between fatigue and control sessions in both experiments. B. Average performance in the 1-back (blue) and N-back tasks (red) in fatigue and control conditions. Error bars indicate the $95 \%$ confidence interval. $C$. Histogram of $\mathrm{N}$-back difficulty levels used in both experiments.

Figure 3. Subjective evaluations and free model parameters in fatigue (gray bars) and control conditions (white bars) for Experiment $1(A-C)$ and $2(D-F)$. Error bars indicate the $95 \%$ confidence interval. ${ }^{\star} p<.05 .{ }^{* \star} p<.01 .{ }^{\star *} p<.001$.

Figure 4. Relationships between session changes in $\mathrm{N}$-back performance, subjective evaluations and free model parameters for Experiment $1(A-D)$ and Experiment $2(E-H)$. ${ }^{*} p<$ $.05,{ }^{* *} p<.01,{ }^{* * *} p<.001$. 


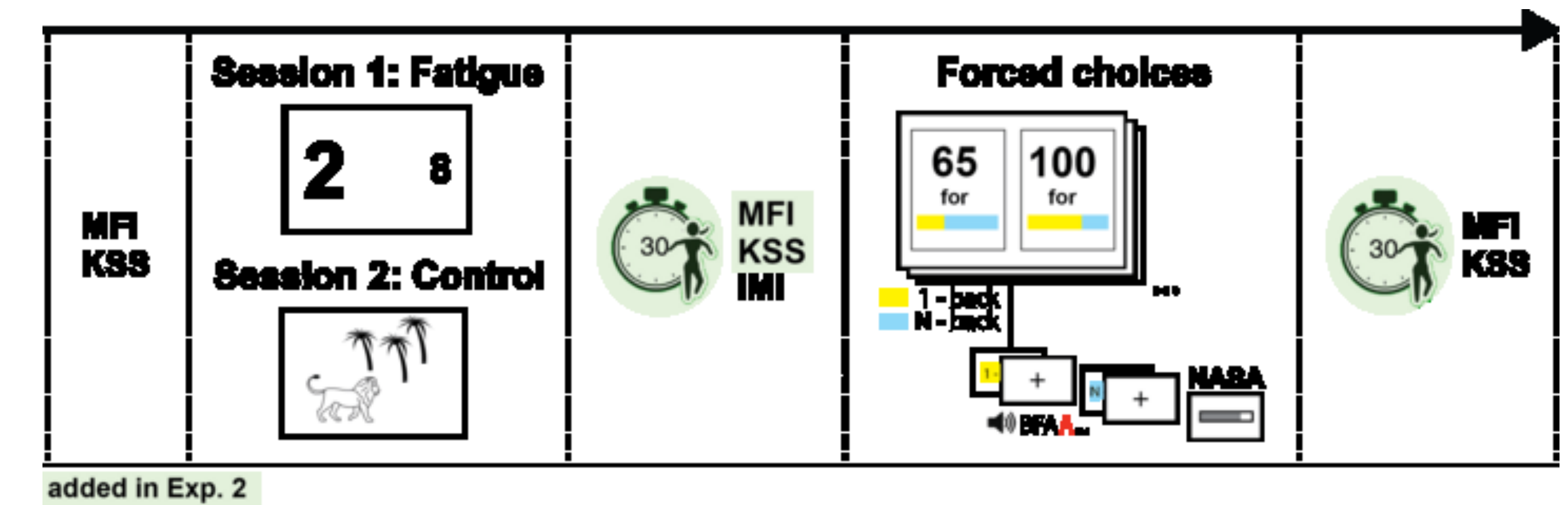

added in Exp. 2 
Exp. 1

A.

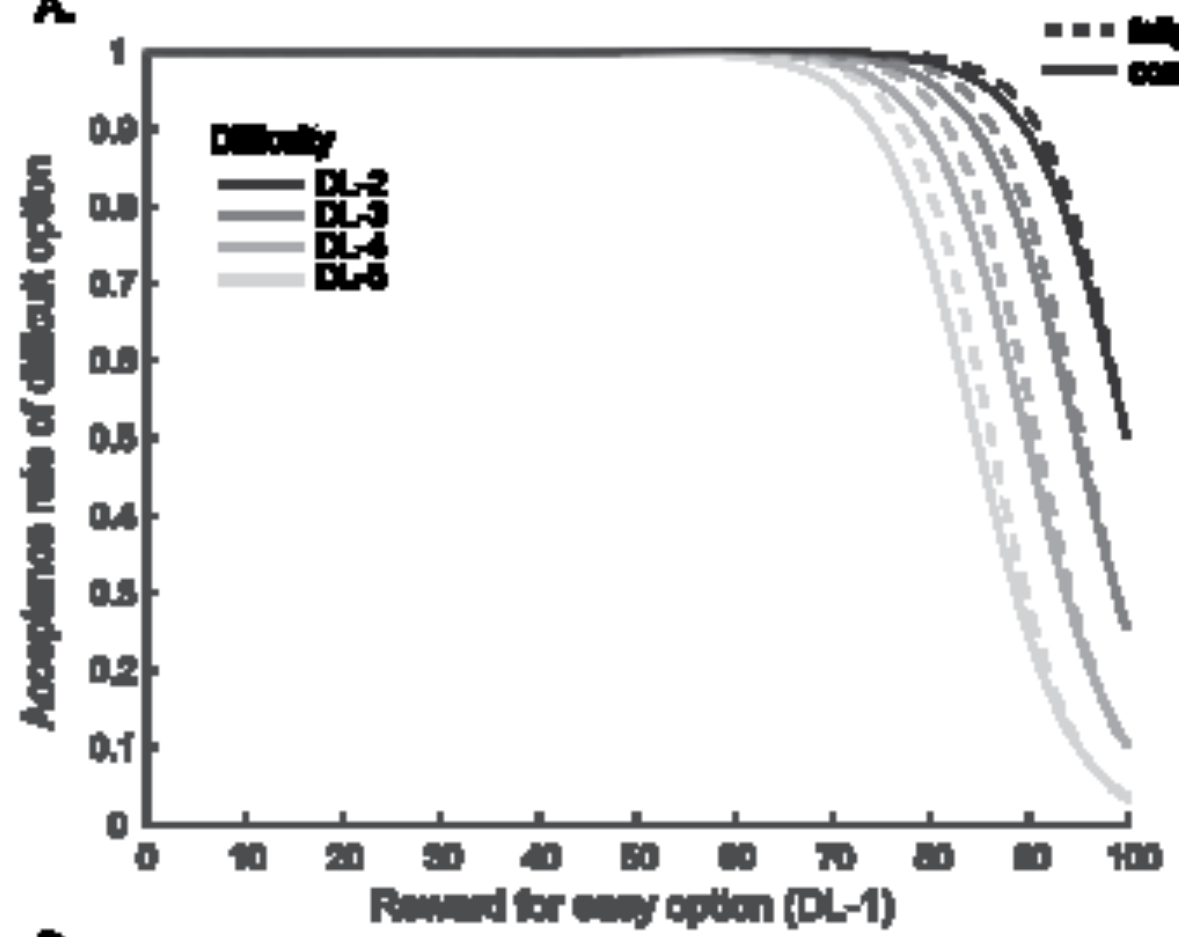

B

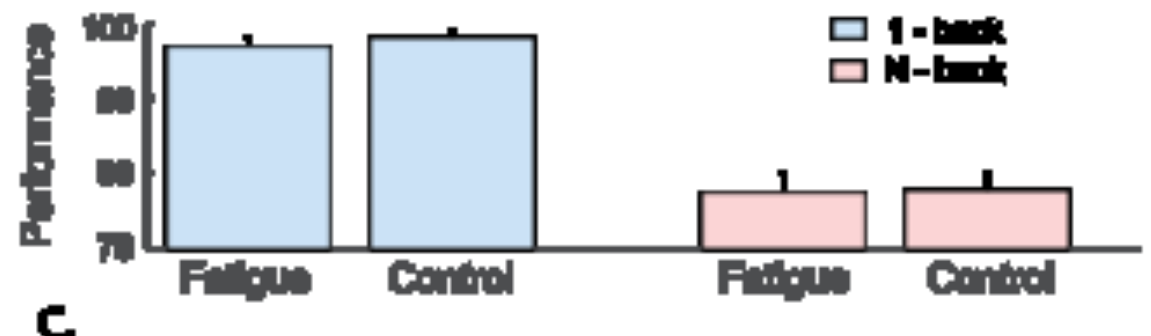

C

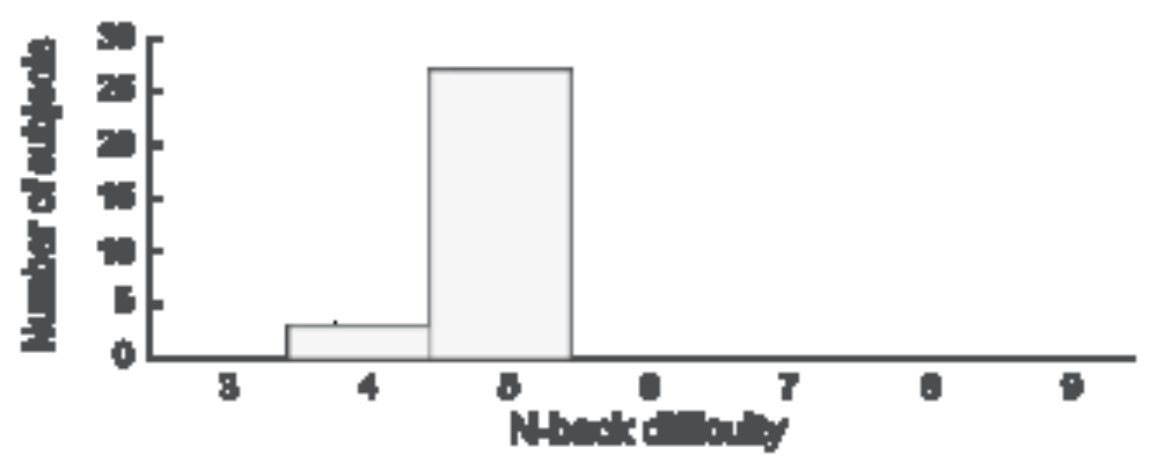

Ep. 2
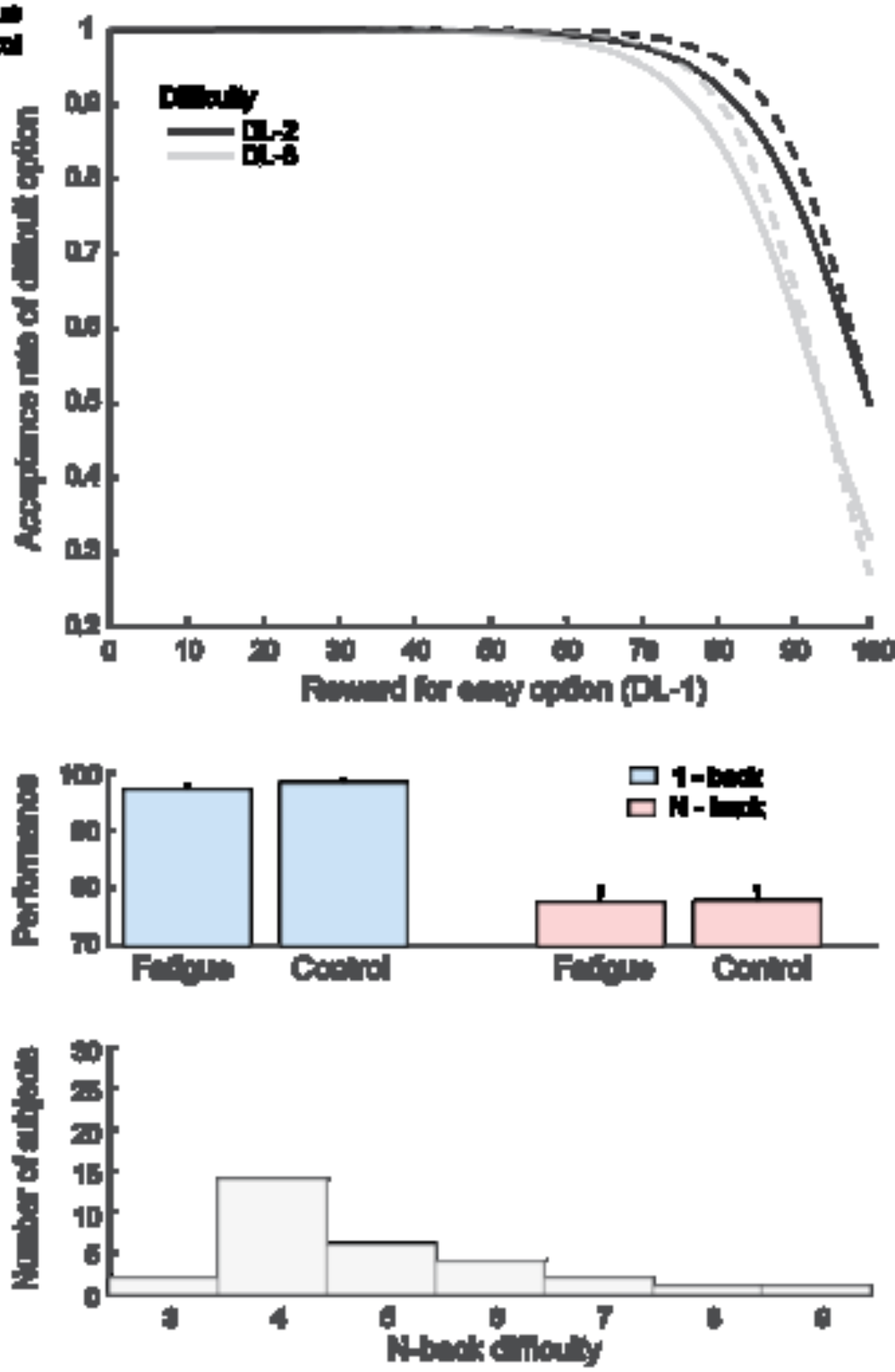
Click here to download high resolution image

\section{Exp. 1}

$\mathbf{A}$

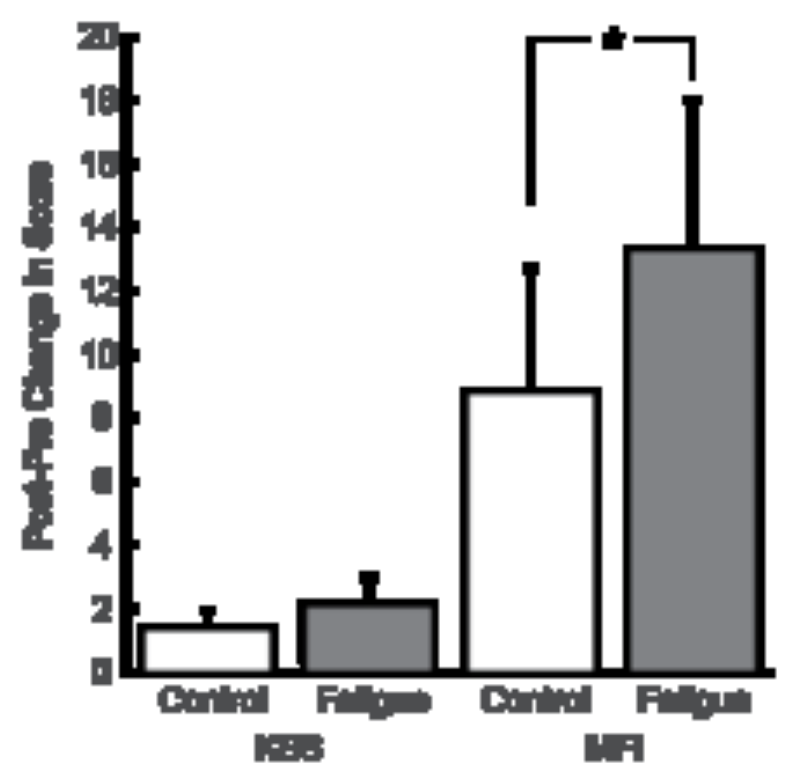

Exp. 2

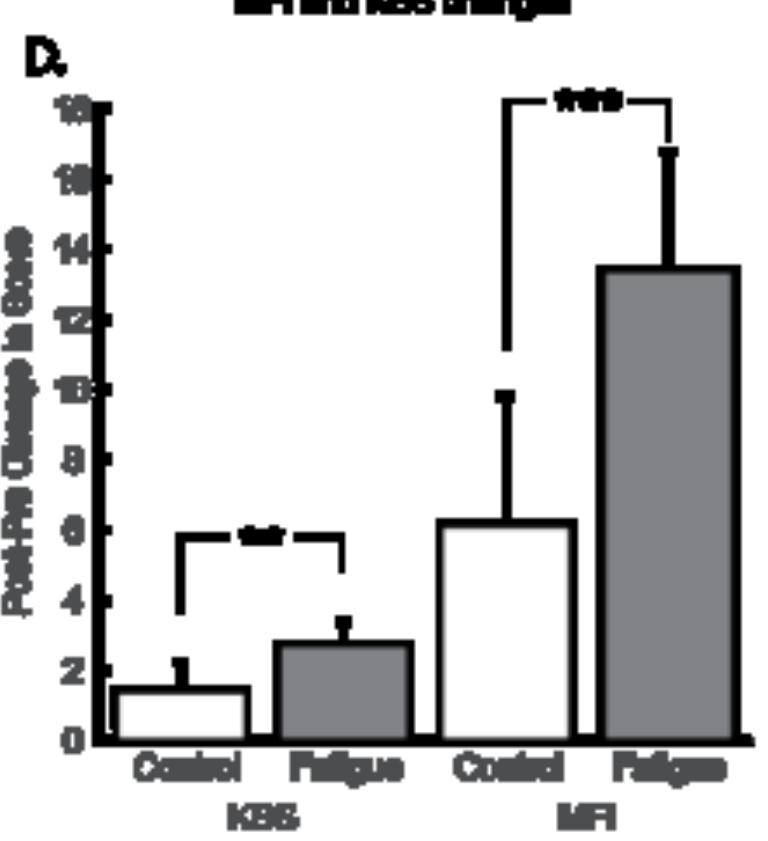

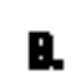

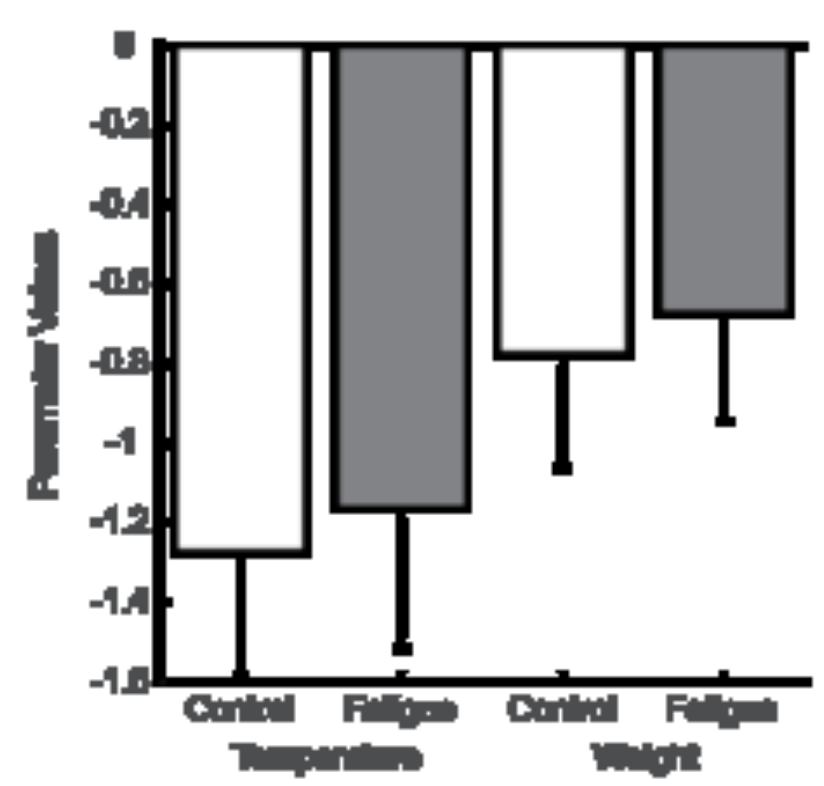

E

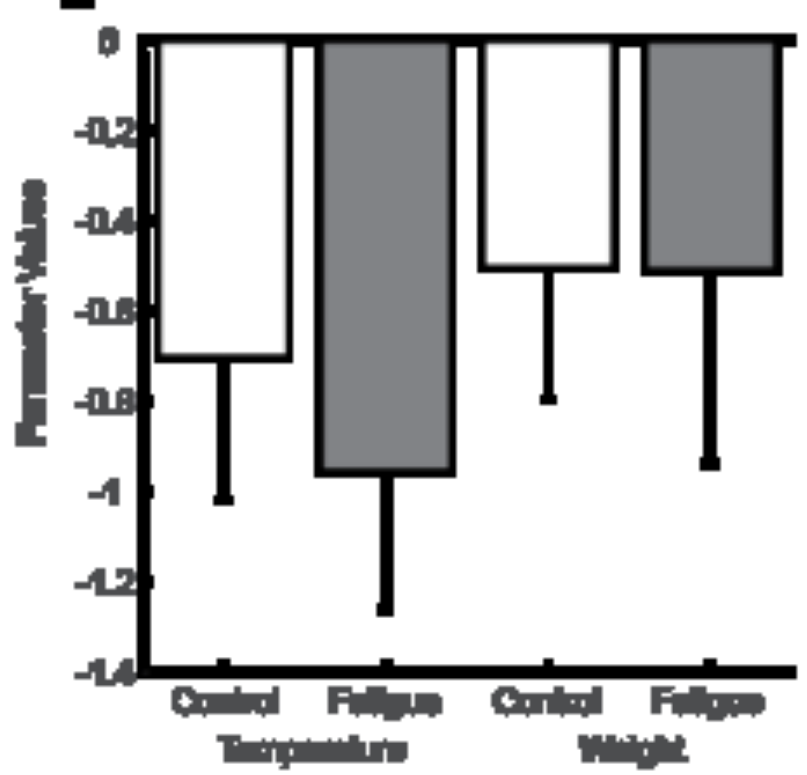

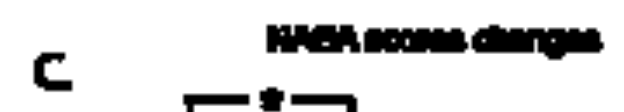

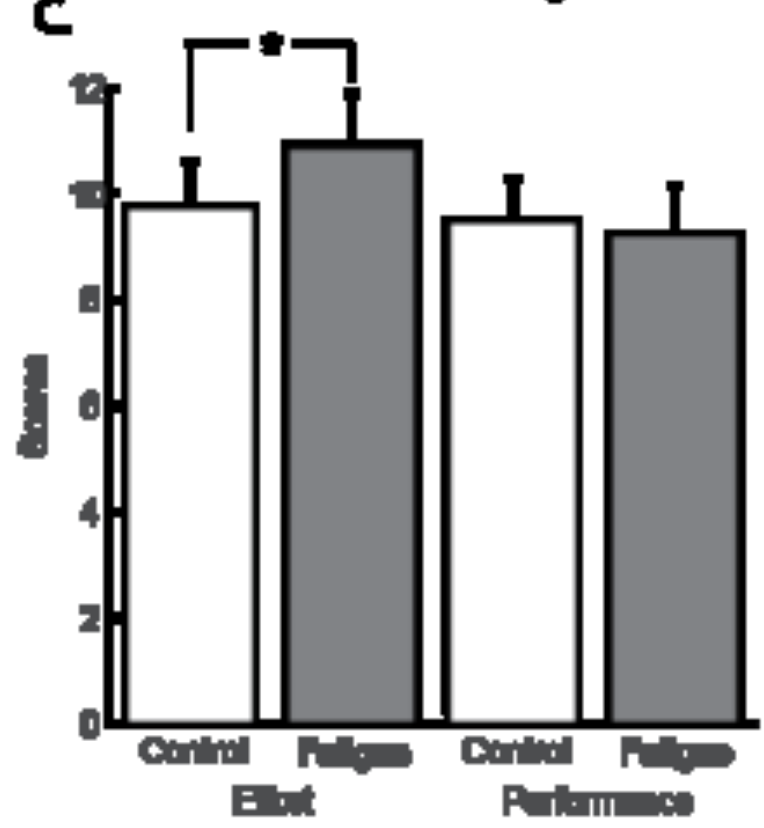

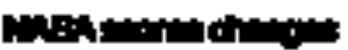

F.

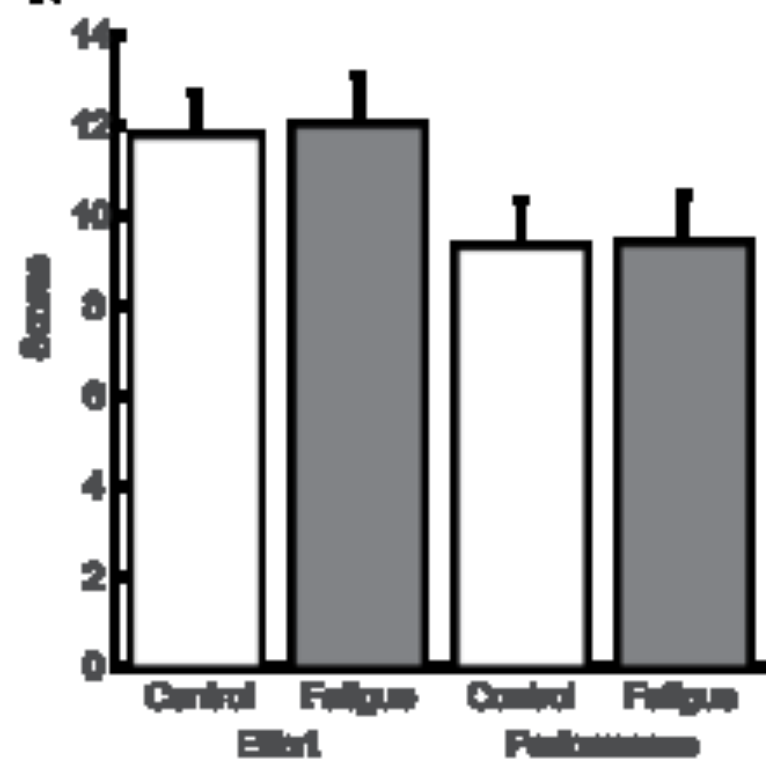


Exp. 1

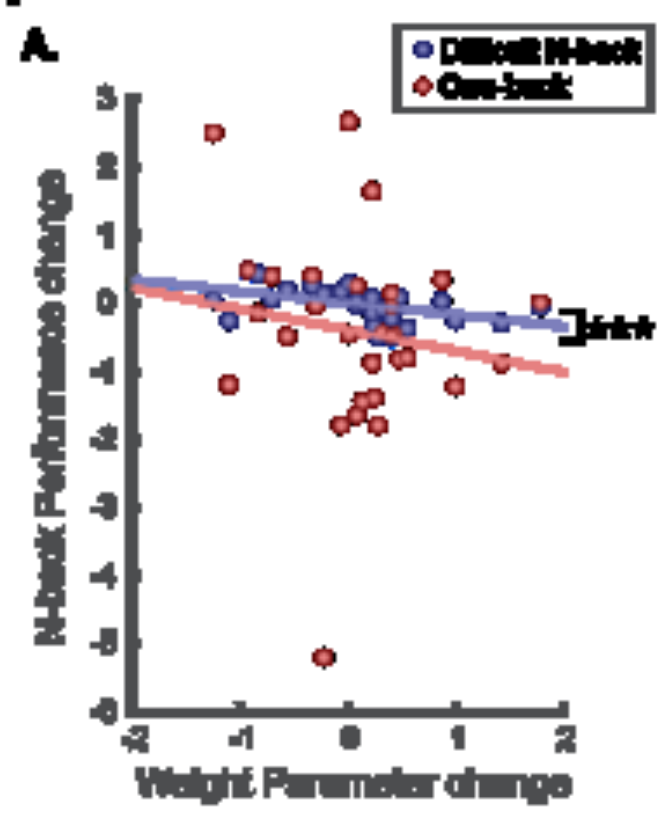

Exp. 2

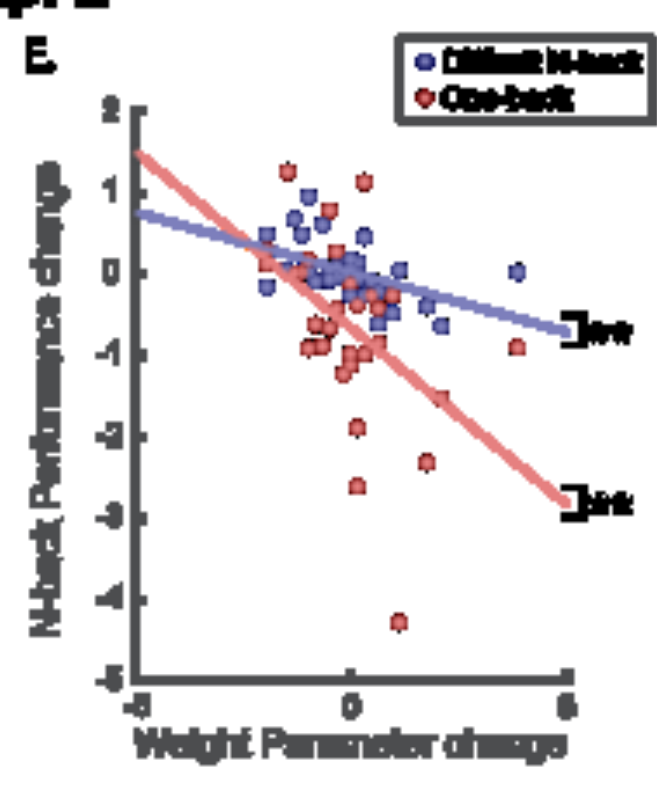

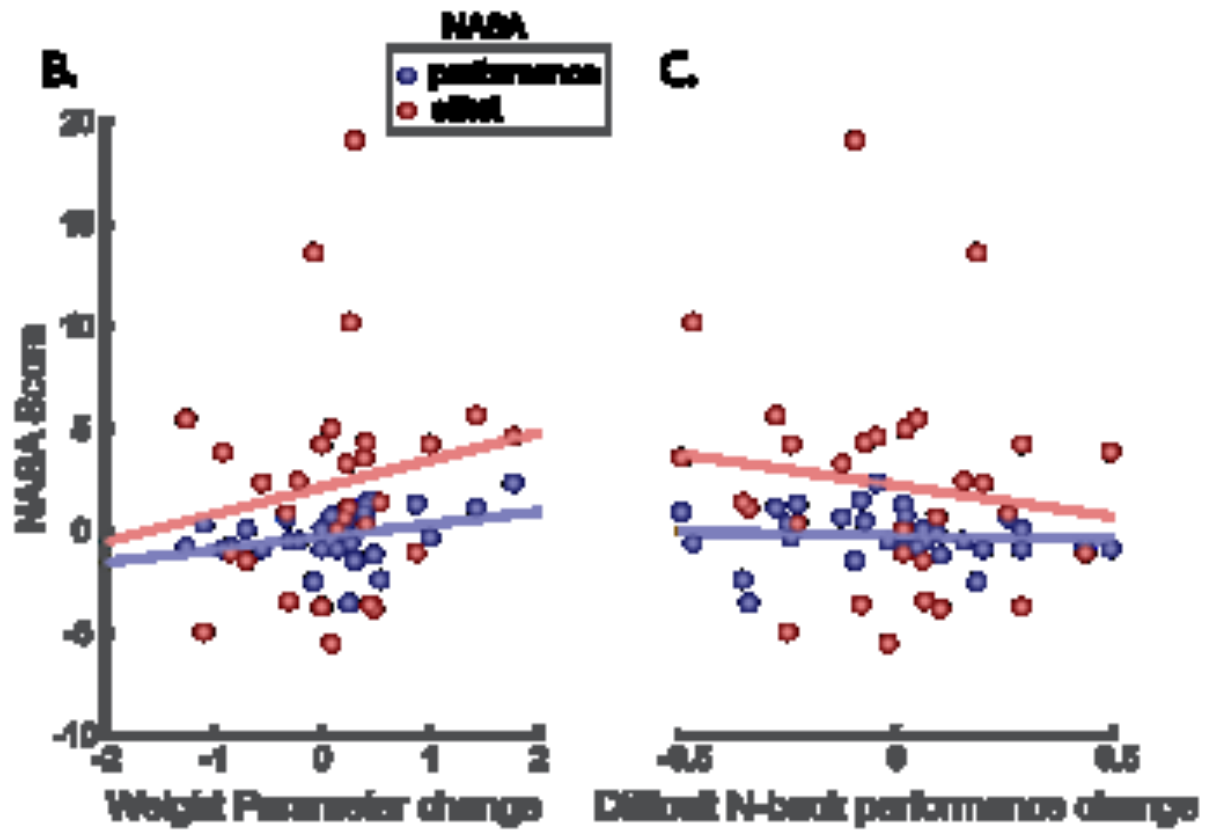

D.
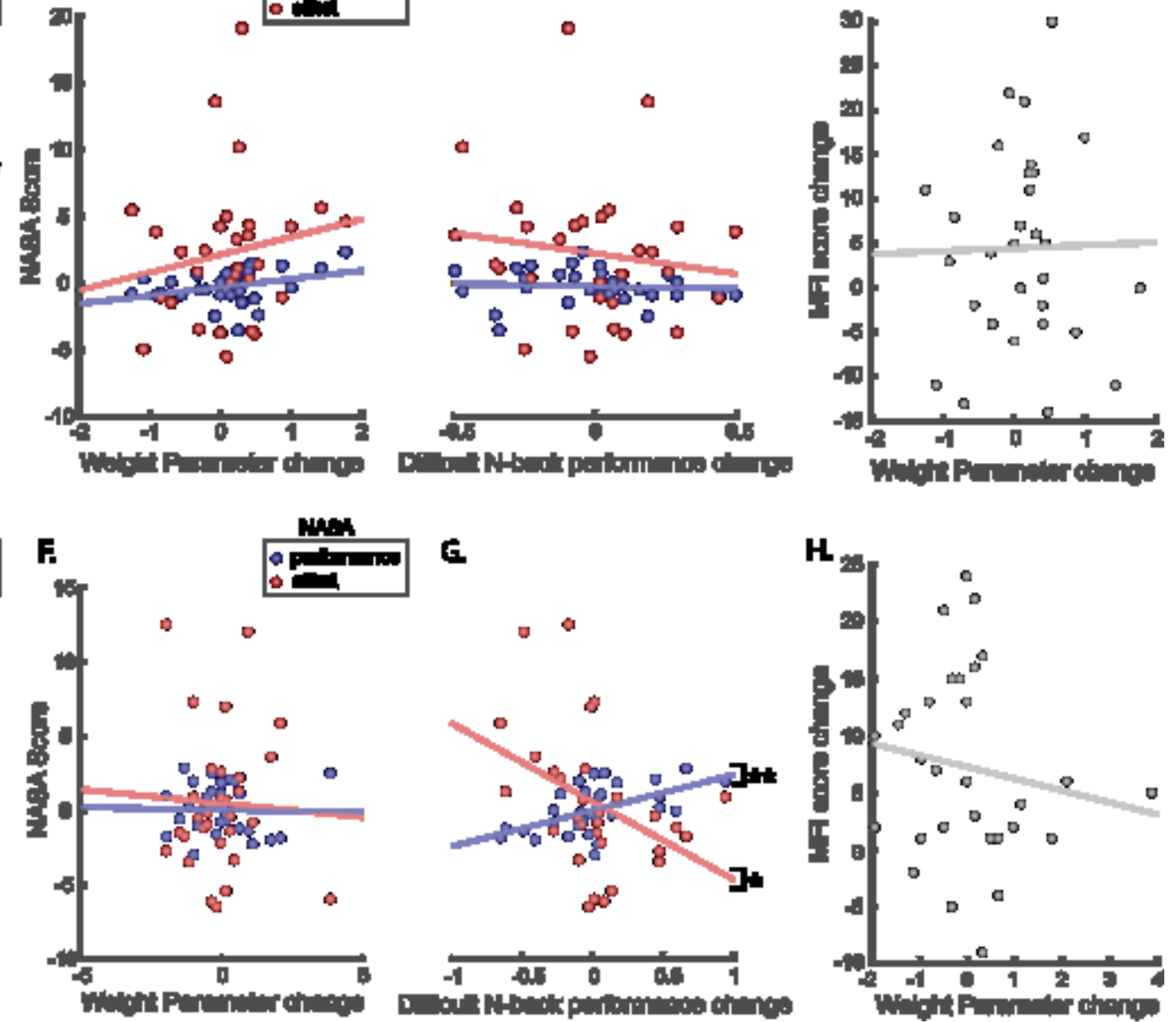

$\mathbf{G}$

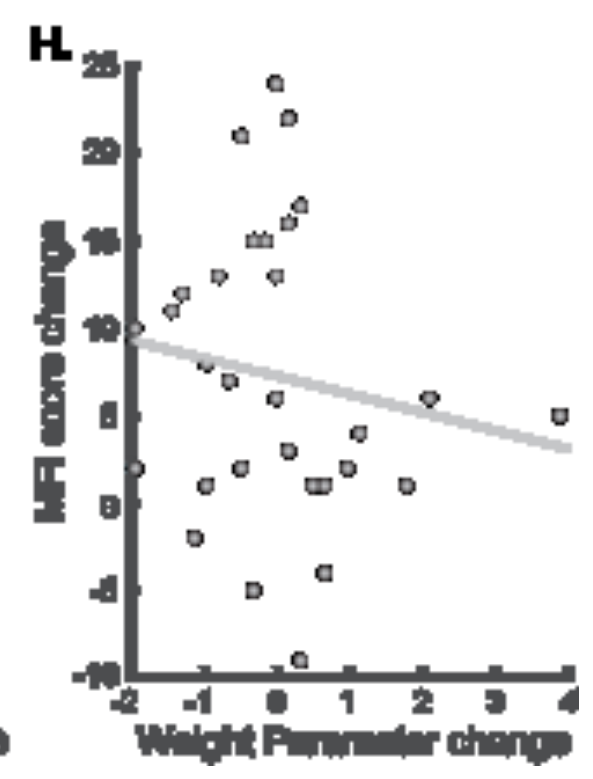

